\title{
DE HARVARD A STANFORD. SOBRE LA HISTORIA DE LA EDUCACIÓN JURÍDIGA EN LOS ESTADOS UNIDOS*
}

\author{
FROM HARVARD TO STANFORD. ON THE HISTORY OF THE LEGAL \\ EDUCATION OF UNITED STATES
}

\section{Rogelio PéRez Perdomo**}

RESUMEN: En la tradición inglesa, la educación jurídica estaba a cargo de la profesión. Esta manera de educar a los abogados no se adaptó bien a la estructura social de los territorios de la América del Norte que formaron luego los Estados Unidos. Desde final del siglo XVIII se introdujo la educación jurídica en las universidades o en escuelas profesionales. El estilo de educación jurídica de los Estados Unidos que conocemos hoy fue creado por Harvard a partir de 1870. Stanford comenzó ofreciendo educación jurídica desde finales del siglo XIX, y se ha transformado en una escuela especialmente reconocida hoy por la innovación y la excelencia. El artículo ofrece una historia corta de la educación jurídica centrada en la contribución de esas dos grandes escuelas.

Palabras clave: Historia de la educación jurídica en los Estados Unidos, Escuela de Derecho de Harvard, Escuela de Derecho de Stanford.
ABSTRACT: In the English tradition, the legal profession was in charge of legal education. This way of educating lawyers was not well adapted to North American society. There, law schools began educating lawyers in late eighteenth century, initially in the hands of proprietary lawyers and later as part of universities. Harvard University invented the present day style of American legal education in the 1870s and Harvard Law School dominated the panorama of legal education until the 1980s. Stanford University started in late nineteenth century and has become a leading institution due to its innovation and excellence. The article offers a short history of legal education in United States centered in these two institutions.

Keywords: History of legal education in U.S, Harvard Law School, Stanford Law School.

* Artículo recibido el 9 de septiembre de 2017 y aceptado para su publicación el 30 de noviembre de 2017.

* Universidad Metropolitana, Caracas, Individuo de Número, Academia Nacional de la Historia, Venezuela.

Boletín Mexicano de Derecho Comparado, nueva serie, año L, núm. 151, enero-abril de 2018, pp. 313-358. 
SUMARIO: I. Introducción: la educación jurídica en la tradición del common law. II. Harvardy su papel en la educación jurídica de los Estados Unidos. III. Stanford: historia y renovación. IV. Allá y aquí. V. Referencias bibliográficas.

\section{INTRODUCGIÓN: LA EDUCACIÓN JURÍDICA EN LA TRADICIÓN DEL COMMON LAW}

La educación jurídica formalmente tal, provista por un departamento o escuela universitaria, tiene una historia corta en los Estados Unidos. Básicamente abarca desde mediados del siglo XIX al presente, un periodo que no llega todavía a los doscientos años. A pesar de su juventud, las escuelas de derecho de los Estados Unidos han adquirido un prestigio mundial en los últimos cincuenta años, y hoy son numerosos los graduados en derecho de Europa, América Latina y demás continentes que consideran útil completar sus estudios jurídicos en los Estados Unidos. Por esto cabe preguntarse cómo se formaron esas instituciones y qué han hecho bien, que ha atraído la atención del resto de los juristas del mundo.

Existe un consenso entre los historiadores del derecho ${ }^{1}$ en cuanto a que la Escuela de Derecho de la Universidad de Harvard tuvo un papel clave como institución, que se convirtió en modelo y dominó intelectualmente el panorama de la educación jurídica en Estados Unidos desde finales del siglo XIX hasta mediados del siglo XX, por lo menos. Por la vía del prestigio institucional impuso los rasgos fundamentales que perduran hasta hoy: el derecho como enseñanza universitaria con una duración de tres años luego de estudios universitarios previos y uso del método de casos. Sus graduados tuvieron una rápida aceptación entre los profesionales, y con frecuencia se convirtieron en profesores en otras universidades y en los miembros de las firmas de abogados más prestigiosas y grandes. El más reconocido historiador de la educación jurídica de los Estados Unidos todavía constataba el predominio de Harvard en la década de $1980 .^{2}$ Sin duda, Harvard sigue teniendo una gran Escuela de Derecho, pero tal vez

1 Friedman, Lawrence M., A History of American Law, 3a. ed., New York, Simon \& Schuster, 2005; Stevens, Robert, Law School. Legal Education in America from 1850s to the 1980s, Chapel Hill, The University of North Carolina Press, 1983; LaPiana, William P., Logic and Experience: The Origins of Modern American Legal Education, New York, Oxford University Press, 1994

2 Stevens, Robert, op. cit., p. XV.

Esta obra está bajo una Licencia Creative Commons

Atribución-NoComercial-SinDerivar 4.0 Internacional, IIJ-UNAM.

Boletín Mexicano de Derecho Comparado, núm. 151, pp. 313-358. 
no tiene el liderazgo de antaño en educación jurídica. Una conocida clasificación de las escuelas de derecho de los Estados Unidos coloca a Yale en primer lugar, y Harvard y Stanford se disputan el segundo lugar con posiciones muy cercanas. En 2016 compartieron el segundo lugar (US Newes and World Report/Education). ${ }^{3}$ Stanford, en cambio, fue una Escuela de Derecho relativamente modesta hasta hace cuarenta años, y ha ascendido con mucha rapidez hasta colocarse entre las primeras y atraer un número importante de graduados y jóvenes académicos del todo el mundo. Seguramente ya no es el tiempo de mirar sólo a Harvard como modelo de educación jurídica en los Estados Unidos.

Estas observaciones preliminares obligan a explicar el título y el enfoque metodológico de este trabajo. La explicación de tomar como punto de inicio a Harvard se ampliará en las páginas que siguen, pero no pretendemos agregar nada a la extensa bibliografía existente sobre la historia de la educación jurídica, incluyendo el excelente trabajo de Pérez Lledó ${ }^{4}$ sobre el tema, uno de los pocos en español. Por lo fundamental, es una revisión de bibliografía. En cambio, la Escuela de Derecho de Stanford ha sido muy poco estudiada. Las fuentes primarias son abundantes, pero han sido poco exploradas. ${ }^{5}$ Este trabajo está basado en esas fuentes y en entrevistas con profesores que han vivido los cambios experimentados por la escuela en los últimos cuarenta años, y por quienes tienen a su cargo el

3 US Neres and World Report realiza anualmente la clasificación más conocida. Entre 2000 y 2014 la Escuela de Derecho de Harvard aparece en segundo lugar 9 veces, y Stanford 10. En varias ocasiones ambas comparten ese lugar. Yale consistentemente ha sido colocada en primer lugar. Startclass, otra publicación, coloca a Stanford a la cabeza, seguida de Yale y Harvard. Para crítica y efecto de las clasificaciones de las escuelas de derecho: Sauder, Michael \& Lancaster, Ryon, "Do Rankings Matter? The Effects of US News and World Report rankings in the Admission Process in the Law Schools", Law and Society Review, vol. 40, Issue 1, 105-134, 2006.

4 Pérez Lledó, Juan A., "La enseñanza del derecho en los Estados Unidos". Doxa, núm. 12, 1992, pp. 41-93. El artículo fue también publicado en Pérez-Lledó, J. A., La enseñanza del derecho. Dos modelos y una propuesta, Lima, Palestra; Bogotá, Temis, 2006.

5 Una fuente muy importante son los boletines y otras publicaciones destinadas a informar a los estudiantes y egresados sobre la oferta de cursos y profesores que estarán a cargo, y de las innovaciones en la vida de la escuela. Otra fuente son las breves biografías de profesores en obras como Marquis who's who in American Law (1a. ed., Chicago, 1977. Varias ediciones posteriores) o el directorio de profesores de derecho publicado desde 1922 (Assotiation of American Law Schools: Directory of Teachers in Member Schools, disponible en: http://heinonline.org/HOL/Index?index=aals/aalsdlt\&́gollections=aals).

Esta obra está bajo una Licencia Creative Commons Atribución-NoComercial-SinDerivar 4.0 Internacional, IIJ-UNAM. Boletín Mexicano de Derecho Comparado, núm. 151, pp. 313-358. 
desarrollo presente de la escuela. ${ }^{6}$ Esta información ha sido recogida con el propósito de analizar qué ha hecho la Escuela de Derecho de Stanford para alcanzar el lugar que hoy tiene y haber pasado a ser un polo de atención mundial.

Las escuelas de derecho tanto de Harvard como de Stanford son parte de universidades, derivan su prestigio de las universidades que forman parte, y a la vez contribuyen a ese prestigio. Harvard es la universidad más antigua de los Estados Unidos, fundada en 1636. Por impulso del presidente (rector) Charles Eliot inició una gran reforma de la educación superior en los Estados Unidos en 1869, y consolidó su proyección mundial en la primera mitad del siglo XX. ${ }^{7}$ La Escuela de Derecho fue una de las protagonistas de esta gran reforma.

La Universidad de Stanford fue creada a final del siglo XIX, y es especialmente en los últimos treinta años cuando se ha hecho conocida en el resto del mundo. Está situada en el llamado Silicón Valley, el centro de la innovación tecnológica, la cultura de lo digital y de la biotecnología, desde final del siglo XX. La universidad misma ha tenido un papel importante en la innovación, y se la ha destacado como centro de creación intelectual. Un artículo de primera página del New York Times que destacaba a Harvard como Stanford del Este (Pérez-Peña, 2014) desató

6 Agradezco a la decana Elizabeth Magill, a los antiguos decanos Paul Brest y Thomas Ehrlich, y a los profesores Lawrence Friedman, Marc Kelman, William Gould IV y Miguel Méndez por las entrevistas que me concedieron. Agradezco también al profesor de Wisconsin, Stewart Macaulay, una larga carta en que me contaba su experiencia como estudiante de derecho en Stanford en la década de 1950. Conversé informalmente con varios otros profesores sobre sus estrategias educativas y sobre su percepción de la Escuela de Derecho y la universidad. Este trabajo no hubiera sido posible sin la colaboración de Sarah Wilson, quien estuvo encargada del archivo de la Escuela de Derecho, y quien me facilitó un riquísimo material sobre la historia de la Escuela de Derecho de Stanford. El personal de la biblioteca de derecho, especialmente B. Williams, S. Stone, A. Holgado y S. Moss, me prestaron también gran ayuda. No puedo dejar de invocar la memoria de John H. Merryman, profesor en Stanford por más de cincuenta años, quien en muchas conversaciones informales me animó a escribir este trabajo y fue muy generoso compartiendo sus vivencias.

7 Charles W. Eliot (1834-1926) era miembro de una familia rica de Boston que se interesó en la investigación científica y en la enseñanza. Viajó a Europa, especialmente a Francia y Alemania, donde observó la enseñanza de las ciencias y el funcionamiento de las universidades. Fue presidente de Harvard entre 1869 y 1909. Bajo su presidencia, Harvard pasó de ser una universidad provincial a ser uno de los modelos de universidad de investigación.

Esta obra está bajo una Licencia Creative Commons

Atribución-NoComercial-SinDerivar 4.0 Internacional, IIJ-UNAM.

Boletín Mexicano de Derecho Comparado, núm. 151, pp. 313-358. 
una caja de truenos. ${ }^{8}$ Por supuesto, lo que se diga sobre Harvard o Stanford como universidades no es necesariamente válido para sus escuelas de derecho.

La escogencia de las escuelas de derecho de Harvard y Stanford para este trabajo tiene también que ver con mi biografía intelectual y con la metodología seguida. Conozco bien las dos escuelas de derecho que analizo. Soy graduado de Harvard (LlM 1972), y he sido visiting scholar allí en ocasiones posteriores. Conozco mejor a Stanford. Desde hace veinte años he sido un profesor visitante frecuente de su escuela de derecho, y en 1982 fui profesor visitante de estudios latinoamericanos. Mis estadías en Stanford me han permitido revisar documentos y entrevistar profesores en los dos años que he desarrollado este proyecto. Por ello, mi análisis para el periodo más reciente de la historia de la educación jurídica se centrará en la Escuela de Derecho de Stanford. No me he planteado como pregunta de investigación cuál es la mejor escuela de derecho, y el lector no debe esperar respuesta a una pregunta que no creo que tenga sentido formularse. También se debe a mi limitación personal que no se diga mucho sobre la Escuela de Derecho de Yale, con la cual he tenido menos contacto.

El lector latinoamericano o europeo seguramente se sorprenderá de que este estudio parta de 1870 para la historia de la educación jurídica en los Estados Unidos. Los estudios universitarios de derecho para la formación de profesionales se remontan en Europa al siglo XII, y en América Latina, al siglo XVI. En Inglaterra se establecieron también dos universidades muy importantes con estudios jurídicos desde el siglo XIII. Allí, como en el resto de Europa, se formaron los abogados estudiando el Corpus Iuris Civilis de Justiniano, y se los llamó por esto civil lawyers. Formaron una elite que tenía el privilegio de litigar en los tribunales de la Iglesia, en el tribunal llamado de Chancellery y en el de Almirantazgo. Pero había otros abogados que se formaban por la profesión misma y no requerían estudios jurídicos universitarios. Litigaban en los tribunales reales. Se denominaban common lawyers, pues conocían ese derecho consuetudinario y

8 Pérez-Peña, Richard, "To Young Minds of Today, Harvard is the Stanford of the East”, New York Times, 29 mayo 2014. Un artículo de Harvard Magazine (sept-oct 2014) comenta el tema, destacando que Harvard sigue siendo una excelente universidad, pero concluye con lenguaje de jóvenes: "Is Harvard (still) cool? Sure. But Stanford is hot". 
jurisprudencial denominado common law. Como consecuencia de la Revolución inglesa, los civil lawyers, que apoyaron al rey en contra del Parlamento, perdieron sus privilegios y terminaron desapareciendo. ${ }^{9}$ De allí viene la distinción entre los países de la tradición del common law (Inglaterra y sus antiguas colonias) y los de tradición del civil law o romano-canónica, especialmente Europa continental y América Latina. ${ }^{10}$ Esta distinción se convirtió en una especie de summa divisio en la comparación de derechos. La diferente importancia de las universidades en la formación de los profesionales del derecho fue uno de los caracteres distintivos de esas dos tradiciones.

La colonización inglesa de la América comenzó unos cien años después que la española, y no todas las instituciones del common law pudieron ser trasplantadas. En particular, la existencia de dos ramas de la profesión con estatus diferentes (barristers y sollicitors) y las instituciones que permitían la educación y organización de los barristers (las inns of courts) no pudieron ser trasplantadas a una sociedad menos jerárquica y que carecía de una aristocracia o clase ociosa. Sin mayores cuadros institucionales, la educación por aprendizaje en la oficina de un abogado dependía mucho del carácter y disposición del abogado. Los testimonios recogidos en Warren ${ }^{11}$ (1912) sugieren que esa educación era básicamente lecturas independientes de obras como la de Coke o Blackstone, ${ }^{12}$ sin mayor input del abogado, y lo que se aprendía transcribiendo documentos en una época en que todo se escribía a mano.

9 Levack, Brian, The Civil Lawyers in England, 1603-1641: a Political Study, Oxford, Clarendon Press, 1973.

10 Merryman, John Henry \& Pérez Perdomo, Rogelio, The Civil Law Tradition, 3a. ed., Stanford, Stanford University Press. 2007.

11 Warren, Charles, A History of American Bar, London, Cambridge University Press, 1912.

12 Edward Coke (1552-1634), abogado, parlamentario y juez de enorme importancia en la política inglesa de su tiempo. Su obra maestra: Institutes of Lawes of England, en cuatro tomos. El primero, publicado en 1628. William Blackstone (1723-1780), jurista inglés que se graduó como civil lawyer en Oxford y luego se hizo barrister. Designado como el primer profesor de derecho inglés en Oxford. Las conferencias que ofreció allí fueron la base de varias publicaciones, que culminaron en sus Commentaries on the Lawes of England (1766). Esta obra fue fundamental para la educación de los juristas en las colonias (luego los Estados Unidos), donde se convirtió en un libro de autoridad. Su éxito fue menor en Inglaterra (Prest, Wilfrid: William Blackstone: Law and Letters in the Eighteen Century, Oxford, Oxford University Press, 2008).

Esta obra está bajo una Licencia Creative Commons

Atribución-NoComercial-SinDerivar 4.0 Internacional, IIJ-UNAM.

Boletín Mexicano de Derecho Comparado, núm. 151, pp. 313-358. 
Como veremos luego, las escuelas universitarias de derecho fueron un trasplante de una institución del continente europeo a los Estados Unidos, y sólo un siglo después la formación de abogados en universidades fue recibida de nuevo en Inglaterra. Por supuesto, había abogados en los Estados Unidos en el periodo colonial. Jugaron un papel muy importante tanto en la independencia como en la primera mitad del siglo XIX. ${ }^{13}$ Por esto, en la primera parte analizaremos por qué se crearon las escuelas de derecho, el impacto que tuvo la de Harvard en la educación jurídica de los Estados Unidos, y también la crítica que generó el método de casos, la innovación harvardiana por excelencia. La segunda parte está dedicada a la Escuela de Derecho de Stanford. Abordaremos brevemente su historia y el contexto en el cual se desarrolló, y cuáles son las innovaciones en la educación jurídica implementadas allí en los últimos años.

\section{HARVARD Y SU PAPEL EN LA EDUCACIÓN JURÍDICA DE LOS ESTADOS UNIDOS}

\section{Educación jurídica en la Colonia y temprano siglo XIX}

En el periodo colonial tardío, en los Estados Unidos había enseñanza de derecho en las universidades, pero no un currículo específicamente jurídico. El primer profesor de derecho fue George Wythe, designado en 1779 en el College William and Mary de Virginia. Entre sus estudiantes estuvieron Thomas Jefferson (luego presidente de los Estados Unidos) y John Marshall (luego presidente de la Corte Suprema). Los estudios de derecho en la universidad no tuvieron buena acogida. Eran percibidos como parte de estudios generales o preparación para la vida cívica ${ }^{14}$ más que útiles para la práctica de la profesión. Fundamentalmente eran cursos con clases magistrales o conferencias. La educación de Jefferson es un buen ejemplo del predominio del aprendizaje sobre la enseñanza universitaria. Wythe era también un abogado eminente, y Jefferson fue su estudiante, y luego fue a trabajar con él como aprendiz. Lo hizo leer los grandes libros de derecho

13 Rehnquist, William, "The Lawyer-Statesman in American History", Harvard fournal of Law and Policy, 9, Issue 3, 1986, pp. 537-558; Tocqueville, Alexis de, La démocratie en Amérique (1835-1840), Paris, J. Vrin, 1990.

14 Warren, Charles, A History of American Bar, London, Cambridge University Press, 1912, p. 341; Friedman, Lawrence M., A History of American Law, cit., 3a. ed., p. 240. 
y lo inició en la práctica jurídica. ${ }^{15}$ Una formación jurídica en el mismo estilo puede apreciarse en John Adams, otro de los padres fundadores de los Estados Unidos. ${ }^{16}$

La habilitación profesional era otorgada por un juez, quien confirmaba que el candidato tenía una buena formación y reputación. El requisito era un periodo de aprendizaje de varios años, pero se apreciaba que el candidato tuviera una cultura universitaria. Esto al menos en las áreas con centros urbanos de importancia. En áreas de menor población, con menos abogados disponibles, las exigencias eran menores. Doce de las trece colonias que formaron luego los Estados Unidos tenían ese requisito del aprendizaje. ${ }^{17}$ La independencia y la forma radical como los principios del laissez faire fueron entendidos hicieron que los requisitos se debilitaran y que dejara de exigirse el requisito de aprendizaje en la primera mitad del siglo XIX. En 1800 todavía catorce de las diecinueve jurisdicciones exigían un periodo de aprendizaje. En 1840 lo requerían once de treinta, y en 1860 , nueve de $39 .{ }^{18}$

A pesar del debilitamiento de los requisitos para la formación jurídica, los abogados mantuvieron un prestigio social importante. Llevaron a los tribunales los conflictos que surgieron en un país recién independizado, y los jueces, que en la tradición del common law se ven como parte del mismo gremio e independientes del gobierno, tomaron decisiones muy importantes en la configuración de la nueva sociedad. Un observador agudo como Tocqueville ${ }^{19}$ destacó tempranamente la importancia de jueces y abogados en la sociedad de los Estados Unidos. Notó que ocupaban el lugar social que correspondía en Europa a los aristócratas, y que eran centrales tanto en el gobierno como en la vida social de esta nueva democracia. Hurst ${ }^{20}$ destacó la creatividad en la liberación de la energía

15 Konig, David T., "Thomas Jefferson and the Practice of Law", Encyclopedia Virginia, Virginia Foundation for the Humanities, Charlottesville, 2012; Bernstein, Richard B., Thomas Fefferson: the Revolution of Ideas, New York, Oxford University Press, 2004.

16 Ferling, John, fohn Adams: A Life, New York, Oxford University Press, 1993.

17 Stevens, Robert, Law School. Legal Education in America from 1850s to the 1980s, Chapel Hill, The University of North Carolina Press, 1983, p. 3.

18 Ibidem, p. 7.

19 Tocqueville, Alexis de, La démocratie en Amérique, 1835-1840, Paris, J. Vrin, 1990.

20 Hurst, William, Law and the Conditions of Freedom in Nineteenth Century United States, Madison, University of Wisconsin Press, 1956.

Esta obra está bajo una Licencia Creative Commons

Atribución-NoComercial-SinDerivar 4.0 Internacional, IIJ-UNAM.

Boletín Mexicano de Derecho Comparado, núm. 151, pp. 313-358. 
del naciente capitalismo. Gilmore ${ }^{21}$ denominó la época como la "edad de descubrimiento". El número de abogados también aumentó rápidamente; en 1850 se estimaba que había 23,939; en 1870, 40,376. ${ }^{22}$

La importancia de abogados y jueces, y las quejas sobre su deficiente formación, hicieron que los jóvenes buscaran maneras de formarse bien en derecho y que algunos abogados establecieran verdaderas escuelas tempranamente. En la bibliografia hoy son llamadas "escuelas de propietarios", pues claramente estaban vinculadas a una persona que enseñaba y cobraba. Estas escuelas no lo eran en el sentido formal que hoy conocemos. No expedían una acreditación, pero otorgaban una carta que certificaba que la persona había cursado ("leído") derecho por un periodo determinado, y esto valía como periodo de aprendizaje. Algunas de estas escuelas llegaron a tener un desarrollo notable. La de mayor éxito fue la de Lichtfield, que llegó a tener 53 estudiantes hacia 1813. En su medio siglo de operación (1780 a 1830) llegó a formar un millar de abogados, un promedio de veinte por año. ${ }^{23}$ Pero, naturalmente, esta educación tan informal no garantizaba mucho.

Las universidades tomaron nota de ese desarrollo y absorbieron algunas escuelas de propietarios o crearon sus propias escuelas. El propósito naturalmente era formar profesionales, pero también dotarlos $-\mathrm{y}$ dotar al derecho - de la pátina de ciencia. En 1860 había ya veintiún escuelas de derecho, entre ellas las que pertenecían a las universidades de Columbia, New York, Pennsylvania y Georgia. ${ }^{24}$

La historia de la Escuela de Derecho de Columbia ha sido muy bien documentada. ${ }^{25}$ Aunque los esfuerzos de establecer una enseñanza de derecho se remontan a mediados del siglo XVIII, el profesor que marcó la temprana historia de la educación jurídica en Columbia fue James Kent (1763-1847). Es importante destacar que él recibió su educación general en Yale, y, conforme a la tradición, fue colocado por su padre como apren-

21 Gilmore, Grant, Ages of American Law, New Haven, Yale University Press, 1977.

22 Stevens, Robert, op. cit., p. 22.

23 Langbein, John H., "Blackstone, Lichtfield and Yale: the founding of Yale Law School", en Kronman, A. T. (ed.), History of the Yale Law School. The Tercentennial Lectures, New Haven, Yale University Press, 2004.

24 Stevens, Robert, op. cit., p. 21.

25 Goebel Jr., Julius, A History of the School of Law, Columbia University, New York, Columbia University Press, 1955. 
diz de Benson, un prominente abogado de Nueva York. Leyó cuidadosamente a Grocio y Puffendord, libros de historia de Inglaterra, incluyendo el de Hume, y una historia del common law. Ya aceptado como abogado, continuó su formación en latín, griego y francés, y leyendo los clásicos. ${ }^{26}$ Fue profesor en Columbia en dos periodos, en 1794-1798 y 1821-1826. Kent básicamente usaba la conferencia como método de enseñanza. Entre 1826 y 1830 publicó sus Commentaires on American Law, una de las obras jurídicas más importantes del siglo XIX en los Estados Unidos. Está constituida por seis partes (en cuatro volúmenes), que cubren (1) el derecho internacional, (2) del gobierno y jurisprudencia constitucional de los Estados Unidos, (3) derecho de los diferentes estados, (4) derecho de las personas, (5) propiedad personal y (6) propiedad de inmuebles. Los comentarios de Kent probablemente nos indican el contenido de su enseñanza, y puede apreciarse que es bastante comprensiva de las distintas áreas del derecho. Los comentarios de Kent se convirtieron en una obra muy apreciada por quienes estudiaban derecho. ${ }^{27}$

A mediados del siglo XIX la Escuela de Derecho más acreditada era la de Columbia, en gran parte por el dinamismo del profesor Theodore Dwight, quien llegó a Columbia en 1858 y enseñó allí hasta $1891 .^{28} \mathrm{El}$ método principal de enseñanza eran las clases del profesor, quien seguía el plan de Blackstone, aunque con más acento en el derecho comercial, y luego la obra de Kent. Además, había sociedades para debates y juicios simulados (moot courts) opcionales. ${ }^{29}$ Columbia, en particular, estableció en 1857 un plan de estudios bastante general, que incluía derecho constitucional, derecho comparado, derecho natural y de gentes, derecho romano, y economía política. ${ }^{30}$

En esa época también empezaron a surgir las firmas de abogados, y los abogados se interesaron en poner obstáculos para el ingreso a la profesión, seguramente con un interés monopolístico, pero también para incre-

26 Goebel Jr., Julius, op. cit., p. 6

27 Ibidem, pp. 23 y ss.

28 Stevens, Robert, op. cit., p. 23.

29 Friedman, Lawrence, A History..., cit., p. 239; McKenna, Marian, Tapping Reeve and the Litchfield Law School, New York, Oceana, 1986.

30 Gordon, Robert W., "The Geological Strata of the Law School Curriculum", Vanderbilt Law Review, 60, 2001, p. 343; Goebel Jr., Julius, op. cit. 
mentar el prestigio de la profesión. En 1890, 23 jurisdicciones exigían un periodo formal de estudio o aprendizaje. ${ }^{31}$

Sin embargo, dentro de la misma profesión no había acuerdo sobre la importancia de la educación universitaria. Había los partidarios de mantener en aprendizaje (es decir, educación por pasantía) y comprobar luego los conocimientos jurídicos con un examen. La universidad era más bien percibida como útil para recibir una educación básica o de estudios liberales. Todavía a comienzos del siglo XX, Thorstein Veblen, profesor muy reconocido como sociólogo, consideraba inapropiado formar abogados en las universidades. Enseñar derecho, decía, era equivalente a enseñar danza. ${ }^{32}$

Tanto por la tradición de que el derecho era un oficio a adquirir por aprendizaje como por la idea de que las universidades debían ofrecer una educación humanística y científica, derecho comenzó como una escuela separada, con requisitos de admisión distintos y más laxos que los requeridos por el college, o cuerpo central de la universidad. Ésta es la situación que encontró Langdell cuando fue llamado primero como profesor y luego como decano por Charles Eliot, recién designado presidente (rector) de Harvard, y que traía como proyecto hacer la educación universitaria a la vez más científica y más útil al progreso del país.

\section{Langdell, método de casos y configuración de la Escuela de Derecho}

Christopher Columbus Langdell (1826-1906) $)^{33}$ es seguramente la persona más influyente en la formación de lo que hoy conocemos como la Escuela de Derecho en los Estados Unidos. Su nombre está indisolublemente asociado a la Escuela de Derecho de Harvard, de la cual fue decano entre 1870 y $1895 .{ }^{34}$ Como ésta alcanzó una indiscutible primacía en la primera mitad del siglo XX, es una especie de padre fundador de lo que hoy es la Escuela de Derecho de los Estados Unidos.

31 Friedman, Lawrence, A History of American..., cit., p. 500.

32 Veblen, citado por Stevens, Robert, Law School, Legal Education in America..., cit., p. 51.

33 Christopher Columbus Langdell provino de una familia poco conocida de New Hampshire; estudió en Harvard en 1854. Fue luego abogado en Nueva York hasta 1870, cuando fue designado profesor, y luego decano. Publicó libros de casos y otros libros apropiados para la práctica del derecho (Kimball, Bruce, The Inception of Modern Professional Education: C.C. Langdell, 1826-1909, Chapel Hill, University of North Carolina Press, 2009).

34 Kimball, Bruce, idem. 
Generalmente se asocia Langdell con la introducción del método de casos en la educación jurídica, que fue inicialmente conocido en los Estados Unidos como "Mr. Langdell's method", ${ }^{35}$ con el inicio de una literatura didáctica asociada con éste, los case books, y con la clase "socrática", en la cual la clase gira sobre las preguntas del profesor y las respuestas de los estudiantes. En los Estados Unidos se destaca como muy importante en el establecimiento del currículo de tres años de estudios de posgrado y con el establecimiento de los exámenes ciegos, en los cuales los profesores no conocen la identidad del estudiante cuando califica sus exámenes. Esto llama menos la atención a quienes han analizado la educación jurídica de los Estados Unidos desde nuestra tradición, ${ }^{36}$ pero no debe ser desatendido. Su lista de asignaturas para el primer año, centrada en contratos, responsabilidad extracontractual, propiedad y procedimiento civil, es decir, en el derecho privado, básicamente logró imponerse en los Estados Unidos. En resumen, Langdell fue un personaje central en la configuración de lo que hoy conocemos como la educación jurídica de los Estados Unidos.

Debe indicarse que en la propuesta de Langdell los estudiantes comenzaban desde su inicio a leer sentencias de tribunales superiores. Era pues una inmersión directa en el derecho positivo, sin curso de introducción ni cursos sobre temas como el derecho romano, la historia del derecho, la filosofía del derecho o el derecho internacional, o sin considerar el cuadro económico o social en que se desenvolvían los casos. La discusión de los casos debía mantenerse estrictamente en el dominio del derecho, pues el propósito era poner en evidencia los principios operativos con los cuales se supone trabaja el profesional del derecho. ${ }^{37}$ Era una educación científico-técnica que postulaba la autonomía del derecho. ${ }^{38}$ No es sorprendente que la propuesta causara muchas objeciones y resistencias, que

\footnotetext{
35 Idem.

36 Pérez Lledó, Juan A., op. cit.

37 Es importante destacar la concepción de ciencia del derecho y de su práctica en Langdell: "El derecho, considerado como ciencia, consiste en ciertos principios o doctrinas. El dominio de esos principios y ser capaz de aplicarlos constantemente con facilidad a los complejos negocios humanos es lo que constituye a un verdadero abogado...". Langdell, citado por Grey, Thomas C., "Langdell's orthodoxy", University of Pittsburgh Law School, 45, 1983, p. 13.

38 Gordon, Robert W., "The Independence of Lawyers", Boston University Law Review, 68, 1988, pp. 1-84; Gordon, Robert W., "The Geological Strata of the Law School Curriculum", Vanderbilt Law Review, 60, 2007, pp. 1245.
} 
vamos a analizar más adelante. Se considera que es hacia 1920 en que se produjo el consenso que el modelo de Harvard era apropiado, ${ }^{39}$ y la influencia de Harvard irradiaba sobre otras universidades.

Sin ningún deseo de disminuir la importancia de Langdell, debe observarse que quienes destacan la novedad y lo peculiarmente americano del método de casos generalmente olvidan o desconocen que los casos se habían usado en la educación jurídica desde Roma, y que la educación por aprendizaje se centra en el estudio de casos. En la tradición inglesa había libros de autoridad, que eran colecciones de decisiones de los tribunales, y se prestó siempre mucha atención a las sentencias de los jueces. Langdell fue un innovador, pero actuó dentro de una tradición. El método de enseñanza fue también de una novedad relativa. A mediados del siglo XIX, las conferencias del profesor que se usaron a comienzos de siglo en las escuelas de propietarios habían sido sustituidas por la recitation: los estudiantes leían antes de clase una obra seleccionada por el profesor, y en la clase el profesor pedía a los estudiantes que repitieran el contenido de lo leído.

En segundo lugar, la renovación de los estudios jurídicos fue parte de una renovación más general de la educación en Harvard y otras universidades, en parte producto del desarrollo de las ciencias en el siglo XIX. En este sentido, el personaje clave es Charles W. Eliot, presidente de Harvard entre 1869 y 1909. Eliot escogió a Langdell como decano, y la gran reforma educativa que protagonizó Harvard no fue sólo de la Escuela de Derecho. Eliot propició la enseñanza de las ciencias en los laboratorios con un trabajo muy cercano de los estudiantes con el profesor. La idea de la universidad como centro de enseñanza de las ciencias y de la aplicación de éstas en la vida real para promover la industria y el bienestar social fueron ideas de Eliot. ${ }^{40}$ Obviamente, Eliot no tenía formación en el derecho, y quien recogió estas ideas y las aplicó al derecho fue el decano Langdell. Eliot, a la vez, sintió que Langdell había reflejado sus propuestas universitarias y sirvió de gran promotor de la Escuela de Derecho, su currículo y metodología. También fue muy importante James Barr Ames (1846-1910),

39 Stevens, Robert, op. cit., p. 41.

40 Chase, Anthony (1979), "The Birth of the Modern Law School", The American Fournal of Legal History, vol 23, núm. 4, pp. 329-348; Touraine, Alain, The Academic System in American Society, New York, McGraw-Hill, 1974. 
uno de los primeros profesores de tiempo completo y totalmente académico en la historia de los Estados Unidos que se convirtió luego en modelo de la ocupación. Ames fue también un profesor que mejoró el método de discusión de casos y logró que éste tuviera aceptación entre los estudiantes. Fue el sucesor de Langdell como decano de Harvard (1895-1910).

La novedad estuvo en la conexión de estas ideas. Langdell se propuso crear un método científico a partir de materiales primarios del derecho, que a su juicio eran las sentencias de los jueces superiores. La idea era extraer y comprender los principios que determinaban las decisiones. En el derecho no es posible un laboratorio donde los estudiantes trabajen con sustancias químicas, plantas o tejidos. En el derecho, el equipamiento fundamental es la biblioteca, y los materiales primarios — según Langdellserían las sentencias de los jueces superiores, convenientemente editadas para que los estudiantes pudieran percibir el hilo del razonamiento y captar los principios que el juez está aplicando. El salón de clase es el laboratorio. La metodología científica requiere que sea el estudiante el que haga el experimento, y que no se limite a ver al profesor haciéndolo. Por esto, el profesor tenía que lograr que el estudiante entendiera el caso y percibiera el razonamiento del juez, poniendo en evidencia sus principios. El papel del profesor no es explicar esos principios, sino lograr que los estudiantes los encuentren gracias a preguntas adecuadamente formuladas. ${ }^{41}$

Su pensamiento estaba claramente influido por el positivismo de Austin. La ambición de lograr una ciencia del derecho independiente de la moral y de los aspectos políticos y sociales que interfieren con el derecho fue frecuente a finales del siglo XIX y comienzos del XX. Lo peculiar de Langdell era su rechazo a la legislación, seguramente muy teñida de opciones políticas que lo disgustaban, así como la concentración en relativamente pocos casos que él escogía para extraer los principios. ${ }^{42}$

Langdell y sus seguidores combinaron la idea positivista del derecho, la ambición cientificista y la tradición de prestar atención a los casos en un método de enseñanza. Pero ésta no fue la única innovación de Harvard,

41 Langdell, Christopher Columbus, "Harvard Celebration Speech", Law Quartely Review, 3, 1887 (consultado en Levine, Martin L. (ed.), Legal Education, New York, New York University Press, 1993).

42 Hacia 1900, el método encontró una sustentación diferente: "The common law is so unsystematic that any study of it as a whole for elementary purposes, we feel, is likely to be followed by little or no accurate or helpful results". Abbott, Nathan, "The Undergraduate Study of Law", Annual Report American Bar Association, 24, 1901, p. 511. 
sino que dio un lugar al derecho en la estructura universitaria. Esto está asociado con la idea de ciencia. De hecho, el derecho sólo tiene cabida en la universidad si es efectivamente una ciencia y no una pura práctica. ${ }^{43}$

\section{Impacto y resistencias}

En Estados Unidos no existía, ni existe todavía, el equivalente de un ministerio de educación que regule los requisitos de ingreso a la Escuela de Derecho o los requisitos para graduarse de abogado. La universidad que existía tenía como propósito una educación general o liberal. Como ya se ha indicado, las primeras escuelas de derecho fueron puramente profesionales, y no estaban vinculadas con la universidad. Cuando a comienzos del siglo XIX las universidades deciden integrar esas escuelas debieron definir su relación con el conjunto de la universidad. Lo que se denominó el "modelo de Harvard" consistía en que los estudiantes debían adquirir primero la formación general que ofrece la universidad, para entrar luego a la Escuela de Derecho. Harvard comenzó a exigir la aprobación del college antes de entrar a la Escuela de Derecho, pensada como una escuela profesional o "graduate", donde los estudiantes se concentraran en el estudio científico del derecho. Esto implica que los estudios jurídicos requieren en definitiva de siete años de estudios universitarios, de los cuales los cuatro primeros no tienen relación con el derecho, pero que deben dar una base cultural y una madurez apropiada para su estudio. Esta exigencia no estaba en ninguna ley ni en las costumbres, y tampoco había alguna autoridad para imponerla. Hacia 1900 todavía la mayor parte de los estudiantes que comenzaban los estudios jurídicos eran graduados en estudios secundarios, y los estudios jurídicos eran paralelos a los del college. ${ }^{44}$ La American Association of Law Schools exigía en 1905 que quienes ingresaran a estudiar derecho tuvieran aprobados los estudios secundarios (high school), y que los estudios jurídicos duraran al menos dos años. Claramente Harvard estaba exigiendo requisitos mayores. ${ }^{45}$

\footnotetext{
43 Langdell, op. cit.

44 Abbott, op. cit., p. 509.

45 "It is for this reason (that the students consider too lengthy to go to a professional school after four years of college) that in part our professional schools are made up largely of non-college students" (Abbott, op. cit., p. 509). Véase también Packer, Herbert \& Thomas Ehrlich, New Directions in Legal Education, New York, McGraw-Hill, 1972.
} 
En 1921, la American Bar Association recomendó que la admisión de un candidato a la barra de los abogados debería requerir estudios completos de college y tres años de estudios profesionales a tiempo completo en una Escuela de Derecho certificada. Fue a partir de esos requisitos de admisión que cada uno de los estados fue aprobando progresivamente como se homogeneizaron, al menos relativamente, los estudios jurídicos en los Estados Unidos. Fue así que a pesar de un eje central de control, los requisitos y el estilo que había impulsado Harvard se convirtieron en estándar para todos los Estados Unidos.

En resumen, el apoyo de la profesión organizada fue muy importante para que la estructura de la educación jurídica propuesta por Harvard se convirtiera en estándar. Se ha entendido que la profesión actuó por razones de mantener su prestigio intelectual, y seguramente también para reducir la competencia (Abel, 1989), pero independientemente de los motivos, la propuesta organizativa y curricular de Harvard marcó la educación jurídica de los Estados Unidos.

El currículo pensado por Langdell también ha tenido una impresionante fortaleza, y constituye lo que Gordon llama el bedrock (eje y estrato más antiguo) del contenido de la educación jurídica estadounidense. ${ }^{46}$

En cambio, la concepción del derecho y el método educativo tuvieron más resistencias desde el principio. La concepción formalista del derecho seguramente ha sido abandonada, y aun cuando algunos rasgos de la metodología educativa han sobrevivido, el campo está abierto a la polémica.

No todos los profesores de derecho de Harvard siguieron la ortodoxia langdelliana, aun bajo el decanato de Langdell. Roscoe Pound fue estudiante de derecho en Harvard en 1889. Más de medio siglo después recordaba a su profesor John Chipman Gray, quien en su libro de casos sobre propiedad comenzaba con referencias al derecho romano. Esto despertó la curiosidad de Pound, quien buscó en la biblioteca una obra de derecho romano. El profesor desaprobó ese texto y le recomendó leer la obra de Sohm (Instituciones de derecho romano) en alemán. ${ }^{47}$ Los recuerdos de Pound nos permiten saber que había más curiosidad intelectual que la permitida

46 Gordon, Robert W., "The Geological Strata of the Law School Curriculum", Vanderbilt Law Review, 60 núm. 2, 2007, pp. 339-370.

47 Pound, Roscoe, "Some Comments on Law Teachers and Law Teaching", fournal of Legal Education, 3, 1951, p. 523.

Esta obra está bajo una Licencia Creative Commons

Atribución-NoComercial-SinDerivar 4.0 Internacional, IIJ-UNAM.

Boletín Mexicano de Derecho Comparado, núm. 151, pp. 313-358. 
por la ortodoxia langdelliana y que había interés por el dominio de literatura extranjera y derechos históricos.

Oliver Wendell Holmes (1841-1935), contemporáneo de Langdell, quien fue brevemente profesor en Harvard, luego magistrado de la Corte Suprema de Justicia de los Estados Unidos, y una de las figuras mayores del pensamiento jurídico de los Estados Unidos, escribió obras que destacan el elemento económico-social y experiencial del derecho. ${ }^{48} \mathrm{O}$, para ponerlo con sus palabras, "la vida del derecho no es lógica, sino experiencia”. Llamó a Langdell un gran teólogo del derecho (Holmes, 1880). Louis Brandeis, quien ingresó a la Escuela de Derecho de Harvard en 1875 y fue luego reconocido como autor, abogado y magistrado de la Corte Suprema de los Estados Unidos, declinó enseñar en Harvard por desacuerdo con las propuestas de Langdell. ${ }^{49}$

Mientras Langdell aun era decano, la revista de Harvard (Harvard Law fournal) publicó artículos de análisis de la legislación y consideraciones sobre las políticas públicas del momento (Grey, 1983). Los llamados realistas americanos (por su oposición al formalismo langdelliano) constituyeron seguramente el pensamiento dominante a mediados de siglo. Generalmente se considera a Holmes dentro de esta tendencia, y se incluye a Llewelyn, Frank, Pound, Oliphant, Moore, Cohen, entre otros. Algunos de ellos enseñaron en Harvard: Roscoe Pound (1870-1964), en particular, fue decano de derecho de Harvard entre 1916 y 1937 y profesor desde 1910. Aun cuando tuvo una relación tormentosa con varios realistas, no se lo puede clasificar como formalista. ${ }^{50}$ De todas maneras, los realistas fueron especialmente fuertes en Yale y Columbia, mucho más que en Harvard..$^{51}$

48 Posner, Richard (ed.), The Essential Holmes, Chicago, University of Chicago Press, 1992.

49 LaPiana, William P., Logic and Experience: The Origins of Modern American Legal Education, New York, Oxford University Press, 1994.

50 En la historia del pensamiento jurídico se destaca a Pound por su propuesta llamada sociological jurisprudence, que de hecho es más cercana a la sociología del derecho que la propuesta de los realistas que destacaban más aspectos psicológicos y psicosociales de las decisiones judiciales. Fue también uno de los promotores del derecho comparado en los Estados Unidos.

51 Kalman, Laura, Legal Realism at Yale 1927-1960, Chapel Hill, University of North Carolina Press, 1986; Gordon, Robert W., "Professors and Policy Makers: Yale Law Faculty in the New Deal and After", A. T. Kronan (ed.), History of the Kale Law School. The Tercentennial Lectures, New Haven, Yale University Press, 2004.

Esta obra está bajo una Licencia Creative Commons Atribución-NoComercial-SinDerivar 4.0 Internacional, IIJ-UNAM. Boletín Mexicano de Derecho Comparado, núm. 151, pp. 313-358. 
Es importante destacar que Yale tuvo una relación dificil con la propuesta de Harvard. Reorganizó su Escuela de Derecho en 1869 y optó por un camino distinto: conectar más el derecho con el resto de la universidad. Esto la orientó a incluir cursos de derecho público y relacionados con la política, mientras que Harvard se orientaba hacia el derecho privado. ${ }^{52}$ Esto explica que Yale fue luego más receptiva con los realistas. Los profesores de Yale tuvieron un papel muy importante en las políticas del New Deal y en los años posteriores. ${ }^{53}$

Los llamados realistas, los critical legal scholars, el movimiento de derecho y sociedad, y derecho y políticas públicas, y, en menor medida derecho y economía, es decir, las distintas tendencias de law and, destacan la importancia del contexto para entender el derecho, y son así opuestos al formalismo. Harvard, por supuesto, no ha sido inmune a estas nuevas maneras de pensar el derecho; pero han sido otras escuelas que las han acogido con más entusiasmo. Yale fue especialmente receptiva al realismo; Wisconsin, a derecho y sociedad; Chicago, a derecho y economía, y Stanford fue hospitalaria tanto a los critical legal studies como al análisis económico del derecho y a derecho y sociedad. ${ }^{54}$

A finales del siglo XX, Ellickson ${ }^{55}$ estudió estadísticamente la producción intelectual publicada en las revistas de derecho. Encontró que todavía la producción doctrinal dominaba, pero los estudios con base empírica habían crecido sustancialmente. Detectó un boom de los estudios críticos, que se desinfló luego, un incremento modesto de law and economics y un creciente número de trabajos sobre normas sociales. Los estudios sociojurídicos, a los cuales corresponden estos últimos, eran más cualitativos que cuantitativos. George ${ }^{56}$ completó el estudio incluyendo los primeros

52 Langbein, John H., "Law School in a University, Yale Distinctive Path in the Late Nineteenth Century", en Kronman, A. T. (ed.), History of the Tale Law School. The Tercentennial Lectures, New Haven, Yale University Press, 2004.

53 Gordon, Robert W., "Professors and Policy Makers, Yale Law Faculty in the New Deal and After", Kronman, A. T. (ed.), History of the Tale Law School. The Tercentennial Lectures, New Haven, Yale University Press, 2004.

54 George, Tracey E., "An Empirical Study of Empirical Legal Scholarship: the Top Law Schools", Indiana Law Fournal, 81, 2006, p. 149.

55 Ellickson, Robert, "The Market for «Law and» in Legal Scholarship", Yale Law School, Faculty Scholarship Series, Paper 377. 1977, disponible en: http://digitalcommons. law.yale.edu/fss_papers.

56 George, Tracey E., op. cit.

Esta obra está bajo una Licencia Creative Commons

Atribución-NoComercial-SinDerivar 4.0 Internacional, IIJ-UNAM.

Boletín Mexicano de Derecho Comparado, núm. 151, pp. 313-358. 
años del siglo XXI, y constató que los estudios empíricos mantenían su crecimiento. Ofreció una clasificación de las universidades, que mostraba cuáles eran las escuelas de derecho que favorecían los estudios interdisciplinarios con uso de estadísticas. Seguramente hoy todavía los estudios doctrinarios son dominantes, en parte por las dificultades de hacer investigación interdisciplinaria, especialmente investigación empírica ${ }^{57}$ pero ya nadie sostiene que se puede hacer ciencia extrayendo principios de las sentencias de los tribunales superiores.

La debilidad de la base teórica propuesta por Langdell obliga a buscar por qué se han mantenido las bases de su currículum y mucho del método que propuso. Ya a comienzos del siglo XX, Ames, el sucesor de Langdell como decano de Harvard, había ofrecido otra justificación al método usado:

Creemos que los hombres entrenados examinando las opiniones de los más grandes jueces que el common law haya producido están en mejor posición de conocer qué es el razonamiento jurídico y probablemente tendrán el poder de solucionar problemas jurídicos mejor que aquellos que se hayan entrenado estudiando las reglas del derecho de un estado en particular. ${ }^{58}$

Esta explicación no es satisfactoria, en el sentido de que no aclara la relación entre la discusión de sentencias judiciales y la capacidad para solucionar problemas jurídicos, y por esto conviene distinguir entre el análisis de casos y la clase, en la cual se le pide al estudiante que analice situaciones de hecho y los principios en los que están basadas las reglas jurídicas invocadas en la decisión judicial. Esta estrategia de discusión progresiva en términos de la dinámica de grupo, ${ }^{59}$ denominada tal vez impropiamente "método socrático", es lo que probablemente desarrolla la capacidad de análisis y síntesis, y a la vez la capacidad de oír y considerar argumentos, que están a la base del razonamiento jurídico. El análisis de casos es sinérgico en la medida en que el trabajo puramente conceptual

57 Schuck, Peter H., "Why don’t Law Professors do more Empirical Research?”, fournal of Legal Education, 39, 1989; Posner, Richard, "Legal Scholarship Today", Stanford Law Review, vol 45, núm. 6, 1993, pp. 1647-1658.

58 Ames, 1907, citado por Stevens, Robert (1983), Law School. Legal Education in America..., cit., p. 56 .

59 Cirigliano, Gustavo y Villaverde, A., Dinámica de grupos y educación. Fundamentos y técnicas, Buenos Aires, Humanitas, 1967. 
o basado en el discurso del profesor internalizado pasivamente por los estudiantes no tiene la misma capacidad de desarrollo del razonamiento dialógico que se requiere en derecho.

En resumen, una crítica fundamental al método langdelliano es también la crítica al formalismo. Al concentrar el análisis en el razonamiento jurídico y en la búsqueda de los principios a los cuales deben referirse las normas específicas, el método lleva a olvidar el elemento experiencial del derecho y su contexto económico, social o su dimensión ética. Los realistas, los critical legal scholars, el análisis económico del derecho y el enfoque sociojurídico llevan a incluir estos elementos. Esto cambia la naturaleza del caso y también el papel del profesor en la clase.

La otra crítica fundamental concierna la clase socrática misma: educación para la jerarquía. En realidad, el profesor langdelliano no intenta construir sobre el conocimiento previo de los estudiantes; por esto no tiene relación con Sócrates, sino sobre la lectura del caso. La dinámica de la clase está dirigida a conducir al estudiante a un principio que el profesor conoce, pero que el estudiante tiene que descubrir. Esto hace la relación muy tensa y lo prepara para aceptar la jerarquía. ${ }^{60}$ Paper Chase (Osborn, 1971) y One L (Turow, 1977) son novelas que se construyen sobre el drama que esto implica para los estudiantes que ingresan en el primer año de derecho en Harvard y en muchas otras escuelas de derecho. ${ }^{61}$ Esto no tiene nada que ver con la personalidad del profesor, que puede ser muy amable y dispuesto a ayudar a los estudiantes fuera del salón de clase.

Estas críticas tuvieron una resonancia limitada, aunque los aspectos más autoritarios y abusivos de la educación jurídica han sido grandemente debilitados, especialmente, como veremos luego, en una Escuela de Derecho como la de Stanford. Pero hay una paradoja en la educación langdelliana si analizamos las competencias que comunica y su relación con el desempeño de los graduados. Los graduados de Harvard y de las otras grandes escuelas que siguieron su liderazgo formaron parte de las grandes

60 Kennedy, Duncan, "How The Law School Fails: A Polemic", Yale Review of Law and Social Action, 1. 1970-1971, pp. 71-91. Véase también del mismo autor, Legal Education and the Reproduction Hierarchy, New York, New York University Press, 2004.

61 La experiencia no era muy diferente en la Escuela de Derecho de Stanford en la década de 1950 conforme una vívida memoria que conserva Stewart Macaulay, quien inició sus estudios jurídicos en Stanford en 1952 (conversación y luego carta personal que me dirigió en 2015, en archivo con el autor).

Esta obra está bajo una Licencia Creative Commons

Atribución-NoComercial-SinDerivar 4.0 Internacional, IIJ-UNAM.

Boletín Mexicano de Derecho Comparado, núm. 151, pp. 313-358. 
firmas de abogados de negocios, se convirtieron en el envidiado tope de la profesión jurídica de los Estados Unidos, y su impacto ha sido mundial en la estratificación de la profesión jurídica. Estos abogados desarrollan una muy buena comprensión de los negocios y son muy buenos en la planificación de las estructuras jurídicas de los negocios. Su mundo es el de las transacciones y contratos, del trabajo con la legislación y las regulaciones que afectan los negocios para sacar el mejor partido de ellas. Pero esto tiene muy poco que ver con la capacidad para analizar sentencias de los tribunales y con el desarrollo del razonamiento jurídico tal como el planteado por Langdell. La educación langdeliana prepararía para el litigio en tribunales superiores, no para las tareas transaccionales de los abogados de negocios.

Una explicación posible es que el impacto sea indirecto: este tipo de educación ejercita la mente en atender a los detalles y a razonar con rigor. Además, selecciona a los mejores estudiantes que han concluido su educación universitaria básica. Esto prepara a la persona para desempeñarse en muchas áreas, y no sólo en el ejercicio de la profesión como abogado de negocios. Sería similar a la persona que se entrena físicamente y luego va a jugar futbol. No ha aprendido a jugar futbol, pero se ha preparado físicamente para aprenderlo rápidamente y convertirse en un buen jugador.

Por supuesto, esto también implica que los recién graduados terminaban de aprender su oficio ayudando a los socios de la firma. En otras palabras, el aprendizaje no fue completamente sustituido por la Escuela de Derecho. Esto fue notado por las firmas, que al tener que adoptar mecanismos exigentes de rendición de cuentas a los clientes comenzaron a exigir que los abogados llegaran mejor preparados para las tareas que debían desempeñar. Esto obligó a las escuelas de derecho a introducir clínicas jurídicas, métodos de simulación, cursos de escritura y legislación, y otras variedades de aprendizaje experiencial. El menú de métodos educativos se ha enriquecido. Ya no puede hablarse de un método en la educación jurídica de los Estados Unidos, sino de una pluralidad de métodos que se usan en ella. Naturalmente, las escuelas de derecho bien dotadas y de tamaño más fácil de manejar han sido muy exitosas en la innovación. De allí el éxito de Yale y Stanford.

El primer año de derecho continúa siendo el baluarte de la herencia langdelliana. Se mantiene su plan de estudios básico, aunque se incorporó el derecho constitucional. El estilo de la discusión en clase es considera- 
blemente uniforme y modelada sobre el estilo de Harvard, ${ }^{62}$ aunque con menos agresividad hacia los estudiantes y más apertura de los profesores. Los libros de casos incorporan con más frecuencia materiales de otras disciplinas, informaciones periodísticas, films y materiales muy diversos. La mayor variedad está en los cursos de segundo y tercer año. El informe de la Fundación Carnegie destaca el éxito del primer año de derecho en comunicar la comprensión y destrezas básicas del razonamiento jurídico, pero a la vez señala su limitación en la comprensión de la complejidad social de los problemas jurídicos y su dimensión ética. ${ }^{63}$

\section{STANFORD: HISTORIA Y RENOVACIÓN}

Existe un material abundante para escribir la historia de la Escuela de Derecho de Stanford, pero acá sólo vamos a proponer una periodización y una caracterización del periodo más reciente. Hay fuentes como la historia para los primeros años de Kirkwood \& Owen $^{64}$ y la memoria de Lempert (2003), que analiza la "clase de 1952", es decir, de quienes ingresaron en 1949 y se graduaron en 1952. Además, existe una abundancia de materiales publicados desde muy temprano en la historia de la Escuela de Derecho. El Announcement of the Departament of Law se inició en 1899 y se convirtió en 1909 en The Law School Annual Announcement. A partir de 1965 se denominó simplemente Stanford School of Law, y desde 1969, Stanford Law School Programs of Study. Desde 1984, el nombre cambió de nuevo a Stanford University Bulletin School of Law, que mantuvo hasta 2014. Desde 1967 se publican los Students Handbooks con información muy detallada sobre la escuela, que puede interesar a los estudiantes. Las cartas que dese 1974 los decanos han dirigido periódicamente a los egresados y al cuerpo profesoral informan de la marcha de la escuela con temas como contratación de profesores, reformas curriculares y marcha de planes de desarrollo. Estas publicaciones tienen una información muy rica sobre quiénes son los profesores, los cursos que se enseñan, el número y procedencia de

62 Mertz, Elizabeth, The Language of the Law School: Learning to "Think Like a Lawyer", Oxford, Oxford University Press, 2007.

63 Sullivan, W. M. et al., Educating Laweyers. Preparation for the Profession of Law, San Francisco, John Wiley, 2007.

64 Kirkwood, Marion A. \& Owens, W. B., A Brief History of Stanford Law School, 18931896, 1961, disponible en: https//wrwe.cdn.law.stanford.edu.

Esta obra está bajo una Licencia Creative Commons

Atribución-NoComercial-SinDerivar 4.0 Internacional, IIJ-UNAM.

Boletín Mexicano de Derecho Comparado, núm. 151, pp. 313-358. 
los estudiantes, las facilidades de que dispone la Escuela de Derecho y, en general, una información de gran utilidad para la historia de la Escuela de Derecho. Aparte de ello, la propia escuela desarrolló un programa de historia oral en la cual los profesores de mayor antigüedad fueron entrevistados por otros colegas o por la archivista de la biblioteca, y conversaron sobre sus recuerdos sobre inicios de su carrera y los cambios percibidos en la Escuela de Derecho. Por último, he conversado con colegas que se graduaron en la Escuela de Derecho de Stanford sobre su experiencia como estudiantes en años diversos, y he entrevistado a un número de profesores y conversado informalmente con un número mayor. Dada la naturaleza de este artículo, este enorme material sólo se usará muy selectivamente.

Las entrevistas realizadas y los documentos de historia oral permiten distinguir tres épocas en la historia de la Escuela de Derecho. La primera, la inicial, va entre su fundación en 1893 y el final de la Segunda Guerra Mundial en 1945. Es una época en que las ambiciones de formar una escuela de derecho de gran calidad encuentran obstáculos muy importantes, que la pusieron en varias ocasiones en peligro de desaparecer. El segundo periodo, también de unos cincuenta años, es de una escuela de importancia creciente, que se consolida como una escuela regional primero, y luego como una escuela nacional muy importante. Por último, el periodo de los últimos veinte años, que puede considerarse la etapa de internacionalización o globalización, en el cual la escuela se ha convertido en un centro de atracción de estudiantes y jóvenes académicos de todo el mundo. La distinción de periodos es puramente instrumental para mostrar los cambios que siempre son más progresivos y complejos. Por ello, lo que haremos es tomar algunos años, que podemos considerar importantes para mostrar las transformaciones que deseamos destacar.

\section{Un dificil comienzo}

La universidad inició sus actividades en 1891 con 555 estudiantes, y fue desde el inicio laica y mixta. Sus fundadores, Leland y Jane Stanford, la establecieron en una enorme granja situada entre San Francisco y San José (todavía es llamada "the Farm"). Para esa época Estados Unidos tenía ya una población considerable (alrededor de setenta millones de habitantes, mayormente concentrados en lo que hoy es el noreste de su territorio), 
con Chicago como la única ciudad importante en el Midwest. California era todavía el Far West que apenas comenzaba su poblamiento. En 1900, California tenía una población de 1.5 millones, y el área de la bahía, donde se encuentra Stanford, unos 650,000. La universidad nació vecina al pequeño poblado de Palo Alto, que era poco más que una estación de tren en 1900 (1,658 habitantes) ${ }^{65}$

Leland Stanford decidió construir una gran universidad en memoria de su único hijo, Leland Stanford jr., muerto cuando apenas contaba quince años. La universidad lleva el nombre del hijo. Stanford había sido gobernador, senador y empresario de líneas férreas: un hombre rico y poderoso. Decidió que la universidad debía ser monumental. Para ello contrató un planificador urbano muy acreditado, Frederick Law Olmsted, y un arquitecto igualmente prestigioso, Henry H. Richardson. El carácter monumental tanto de la planificación como de los edificios se explican por la intención de honrar la memoria del hijo. Consciente del carácter extraurbano, la universidad nació con viviendas para profesores y estudiantes. Esto ha permitido que la gran mayoría de sus estudiantes y profesores desde su inicio hasta hoy sean de tiempo completo; pero también implicó un comienzo difícil. La universidad se inició abierta a personas de todas las clases y gratuita para los estudiantes (lo fue hasta 1920). Sólo cobraba la vivienda y la alimentación.

El contexto en el cual se ha desenvuelto Stanford ha cambiado. California se convirtió en el estado más poblado del país (población estimada: 39 millones en 2015), y Stanford se encuentra en el llamado Silicon Valley, la zona que en el mundo es reconocida por su espíritu de innovación y emprendimiento. La Universidad de Stanford ha sido parte de ese ambiente de innovación, y su desarrollo como una de las principales universidades del país se ha beneficiado de él. En 2015 tenía 7,000 estudiantes de licencia y 9,770 posgraduados (total 16,770 estudiantes) en uno de los campus más grandes de los Estados Unidos. ${ }^{66}$ La mayoría de los estudiantes y profesores viven en el campus o en las pequeñas ciudades que lo rodean (Palo Alto y Menlo Park), aunque algunos prefieran una vida más urbana y residen en San Francisco.

65 Todas las cifras de población son de los censos decenales de los Estados Unidos, y están tomadas de Internet.

66 Stanford University. Common data set 2015-2016. Disponible en: ucomm.stanford.edu.

Esta obra está bajo una Licencia Creative Commons

Atribución-NoComercial-SinDerivar 4.0 Internacional, IIJ-UNAM.

Boletín Mexicano de Derecho Comparado, núm. 151, pp. 313-358. 
Los estudios jurídicos en Stanford comenzaron como parte del college. Derecho era así un departamento, no una escuela profesional. Los propósitos anunciados en 1892 eran muy ambiciosos:

El fin último del departamento cuando esté completamente desarrollado será el de (1) Proveer en los principios elementales del derecho angloamericano que debe formar parte de la formación ciudadana. (2) Proveer instrucción en derecho comercial que pueda ser adecuada para quienes proyecten convertirse en comerciantes, banqueros, o seguir otra línea de negocios. (3) Proveer a los estudiantes que proyecten entrar al servicio público instrucción en derecho público e internacional. (4) Proveer a los estudiantes de ciencias políticas y sociales el entrenamiento en derecho necesario para esas ramas. (5) Ofrecer el estudio de la jurisprudencia como ciencia y, en consecuencia, enseñar historia del derecho e instituciones jurídicas. (6) Proveer a los estudiantes que deseen convertirse en profesionales del derecho un curso completo que les permita ejercer el derecho en cualquiera de los estados o donde el common law de Inglaterra prevalezca. ${ }^{67}$

Como puede apreciarse, el proyecto fue ofrecer los cursos jurídicos como parte de la educación general de la universidad, pero también con la posibilidad de que el estudiante se preparara para la profesión de abogado. Recordemos que todavía a final del siglo XIX se consideraba que el aprendizaje era la mejor forma de preparar a los abogados.

Los estudios jurídicos se iniciaron desde 1893 con dos profesores: Benjamin Harrison, quien venía de ser presidente de los Estados Unidos, ${ }^{68}$ y Nathan Abbott, quien realmente desarrolló los estudios jurídicos y los dirigió hasta 1907. ${ }^{69}$ También enseñó Edward Hamlin Woodruff, ${ }^{70}$ inicialmente contratado como bibliotecario de la universidad.

67 Kirkwood y Owens, op. cit., p. 3. Traducción del autor, con ligera simplificación del texto original.

68 Benjamín Harrison (1833-1901) fue presidente de los Estados Unidos 1889-1893. Fue también un abogado conocido, que entre otros asuntos tuvo la representación de Venezuela en 1900 en su conflicto con Gran Bretaña por el asunto de Guyana (Calhoun, Charles W., Benjamin Harrison, New York, Times Books, 2005).

69 Nathan Abbott (1854-1941). Nativo de Maine, graduado en Yale y luego estudió derecho en Boston College. Ejerció brevemente el derecho y luego fue profesor en Michigan y Northwestern. Fue profesor y director del departamento de derecho en Stanford entre 1895 y 1907. Luego fue profesor en Columbia hasta 1922. Se retiró y murió en California. Tenemos de él sólo dos trabajos sobre educación jurídica. Era muy cuidadoso en su enseñanza y muy apreciado por los estudiantes, además de interesarse en arte, literatura y 
En junio de 1893 murió el senador Stanford y se produjo un litigio, que comprometió a sus bienes. La universidad tenía un patrimonio separado, pero no tenía ingresos suficientes para mantenerse mientras no pudiera entrar en posesión del legado de Stanford. Los profesores debieron aceptar una disminución de sus salarios, y no había dinero para formar una biblioteca. En estas condiciones, el presidente de la Universidad se vio en serias dificultades para contratar profesores para la Escuela de Derecho. Los herederos de Stanford ganaron el juicio en 1896, y la situación financiera de la universidad mejoró. La descripción que hace el profesor Clarke Butler Whittier ${ }^{71}$ de lo que era el departamento de derecho en 1897 muestra su precariedad y pobreza: dos salones de clase y una biblioteca que ocupaba una habitación en un dormitorio. ${ }^{72}$

Hacia 1900 se formó la Asociación de Escuelas de Derecho de América. Stanford fue admitida como uno de los 27 miembros fundadores aun cuando no había graduado a nadie en derecho. Abbott se convirtió muy pronto en presidente de la asociación. Stanford tenía cinco profesores de tiempo completo en derecho, y ofrecía un plan de estudios apropiado para la formación profesional. Todos los profesores usaban el método de casos, y se requería un total de seis años de estudio para el grado de derecho, fijando así un estándar más alto que el común en la época. En 1901, Stanford tuvo su primer graduado en derecho. ${ }^{73}$

En 1908, el departamento de derecho pasó a llamarse Escuela de Derecho, acentuando el carácter profesional que se quería dar a la enseñan-

viajes, según la nota necrológica publicada por Columbia Law Review (Stone, Harlan F., "Nathan Abbott", Columbia Law Review, XLI, núm. 6, 1941, pp. 577 y 578). Su biografía revela que para la época era más atractivo enseñar en Nueva York que en un despoblado de California. Abbott fue llamado a Stanford porque el profesor inicialmente escogido fue Ernest Huffcuff, de Chicago, quien prefirió irse a Cornell cuando recibió oferta de esa universidad.

70 Edward Hamlin Woodruff era graduado en derecho y miembro de la barra de abogados de Nueva York antes de viajar a California para trabajar en Stanford. En 1895, Woodruff se fue a Cornell como profesor de derecho (Kirkwood y Owens, op. cit., pp. 5 y 9).

71 Whittier (1872-1943) fue uno de los primeros graduados de Stanford, y luego se graduó en derecho en Harvard en 1896. En 1901 se fue como profesor a la Universidad de Chicago. Preparó un libro de casos, que se ha reeditado como un clásico en 2012: Cases on Common Law Pleading (1912), y un libro sobre Theory of Pleading (1908). Regresó como profesor a Stanford en 1915, donde se mantuvo hasta su retiro en 1937 (Kirkwood \& Owens, op. cit., p. 59).

72 Kirkwood \& Owens, op. cit., p. 10.

73 Ibidem, pp. 15-17.

Esta obra está bajo una Licencia Creative Commons

Atribución-NoComercial-SinDerivar 4.0 Internacional, IIJ-UNAM.

Boletín Mexicano de Derecho Comparado, núm. 151, pp. 313-358. 
za. En 1919 fue certificada la Escuela de Derecho por la American Bar Association. Desde 1924, la escuela comenzó a exigir el título de pregrado como requisito de ingreso. Los esfuerzos de Stanford por transformarse en una respetable escuela de derecho encontraron enormes obstáculos. El primero, al inicio de su vida, fue la muerte del senador Stanford y el litigio sobre su patrimonio. En 1906 fue el gran terremoto de San Francisco, que destruyó o afectó una parte de los edificios de Stanford. En 1917, Estados Unidos entró en la Primera Guerra Mundial, y una parte de los estudiantes y profesores de la Escuela de Derecho fueron movilizados. En 1940-1945, la movilización más radical para la Segunda Guerra Mundial dejó a la Escuela con relativamente pocos estudiantes y profesores. En 1943 se inscribieron treinta estudiantes. Esto obligó a un crecimiento muy lento de la Escuela de Derecho: en 1912 había siete profesores de tiempo completo; en 1945-1946 había nueve, además de un visiting profesor y siete "lecturers", que en la época eran profesores por hora, que tenían otras ocupaciones. ${ }^{74}$

La Escuela de Derecho tenía dificultad para retener a sus profesores. En la biografía de los profesores que hemos esquisado en notas al pie de página se nota que después de unos años de enseñar en Stanford, y seguramente después que el profesor se hacía más conocido, la tendencia era la migración a las buenas universidades de la costa este. Ni siquiera Nathan Abbott, cuya importancia es capital en la historia de la Escuela de Derecho de Stanford, escapó a esa tendencia, y se fue a Columbia, donde fue profesor hasta su retiro. Frederic C. Woodward, ${ }^{75}$ quien fue designado decano en 1916, renunció al año para irse a Chicago.

\section{La etapa de consolidación y cambio}

Para mostrar lo que significa la consolidación de la Escuela de Derecho Stanford en el periodo 1945-1995, he decidido centrar la atención en dos momentos específicos: 1950 y 1975, y los años cercanos a esa fecha.

\section{Idem.}

75 F. C. Woodward tuvo una carrera distinguida. Graduado en Cornell (BA y MA en derecho, 1894 y 1895) ejerció como abogado en Nueva York. Fue profesor de derecho en Northwestern (1902-1907) y en Stanford desde 1907. Culminó su carrera en Chicago, donde llegó a ser vice-presidente de la Universidad. La carrera de Woodward puede reflejar el prestigio relativo de las escuelas de derecho a comienzos del siglo XX. Publicó varios artículos en derecho penal (Kirkwood \& Owens, op. cit., p. 28).

Esta obra está bajo una Licencia Creative Commons Atribución-NoComercial-SinDerivar 4.0 Internacional, IIJ-UNAM. Boletín Mexicano de Derecho Comparado, núm. 151, pp. 313-358. 
Para los primeros años de la década de 1950, además de las fuentes documentales, se cuenta con el trabajo de Lempert, ${ }^{76}$ quien recogió datos en 1981 con entrevistas a varios de los miembros de la promoción de 1952. Para la década de 1970 contamos con varios testimonios de la historia oral de la Escuela de Derecho y con varias de las entrevistas realizadas para este trabajo.

Hacia 1950, la Escuela de Derecho se había recuperado de la enorme sacudida que significó el retiro o permiso a estudiantes y profesores que fueron movilizados o colaboraron de otras maneras con el esfuerzo bélico de la Segunda Guerra Mundial. Claramente era una escuela de importancia regional. En 1949 aspiraron a ingresar 277 estudiantes, y dada la capacidad física de la escuela se admitieron 150. El grupo era homogéneo en su procedencia geográfica y social. Eran todos blancos, y sólo cinco eran mujeres. Académicamente no eran muy distinguidos: algunos de los admitidos tenían promedios por debajo de C+ -una calificación apenas pasable. ${ }^{77}$ En 1950, la escuela tenía 450 estudiantes, de los cuales aproximadamente el 75\% informaban que su hogar (home residence) estaba en California (Stanford Bulletin 1950-1951). Alrededor de dos tercios de la clase se habían graduado en Stanford u otros colleges de California, y sólo el 10\% venían de Ivy League colleges. ${ }^{78}$ Cuatro de cada cinco habían cursado la secundaria en instituciones públicas. Aproximadamente la mitad de la clase eran veteranos de la Segunda Guerra Mundial. Esto implicaba que el gobierno federal pagaba su matrícula. El profesor Turrentino señalaba que el G.I.Bill (la norma que estableció el pago de matrícula universitaria a los veteranos) había salvado a Stanford, y que la Escuela de Derecho pudo incrementar el salario de los profesores. ${ }^{79}$

Todavía cuando Manning entró como decano en 1964 encontró que los egresados y el cuerpo estudiantil estaban mayormente integrados por californianos, e hizo campaña para atraer estudiantes de otros estados, especialmente en el oeste de los Estados Unidos. ${ }^{80}$

76 Lempert, David, "After Five Decades: Stanford Law School class of 1952”, Legal Studies Forum, 27, 2003.

77 Ibidem, p. 266.

78 Idem.

79 Turrentino, citado por Lempert, op. cit., p. 267.

80 Manning, Bayless, Bayless Manning. Reflections on his Years at Stanford Law School, Interviewed by Sarah F. Wilson, Feb 18, 2008, Oral History Series, Stanford Law School, 2008.

Esta obra está bajo una Licencia Creative Commons

Atribución-NoComercial-SinDerivar 4.0 Internacional, IIJ-UNAM.

Boletín Mexicano de Derecho Comparado, núm. 151, pp. 313-358. 
En 1950, la Escuela de Derecho contaba dieciséis profesores (de los cuales dos asociados y uno asistente) y 28 otras personas listadas como parte del personal de enseñanza, en su mayoría lecturers, ${ }^{81}$ que ejercían la profesión de abogado. Seis de los dieciséis profesores eran graduados en derecho de Harvard, y seis de la propia Stanford. Uno, Car B. Spaeth, tenía un grado en Oxford, y había sido sterling fellow en Yale. Dos habían sido clerks en la Corte Suprema de Justicia de los Estados Unidos, una distinción considerada muy importante en la época, y que servía para iniciar una carrera académica. Es interesante observar que en la breve presentación de profesores se destacan sus servicios en la guerra. La búsqueda realizada hasta ahora muestra que muy pocos tenían publicaciones, lo cual era frecuente en la época: no se esperaba de los profesores creación de conocimiento.

En 1950, los cursos eran los tradicionales: en primer año, contratos, responsabilidad extracontractual, propiedad inmueble, propiedad personal, agency y bibliografía jurídica. En segundo año había ocho cursos obligatorios: derecho constitucional, derecho mercantil, corporations, conveyances (documentos de traslado de propiedad), remedios contractuales, derecho procesal, trusts y escritura jurídica. El enfoque era el derecho de California, pues la mayoría de los estudiantes eran californianos o se esperaba que ejercieran el derecho en California. No había cursos de derecho del trabajo, antitrust, derecho regulatorio (o administrativo), campos que eran novedosos en la época. Se discutió si introducir sociología del derecho, pero se descartó la idea, por considerar que esto no era derecho en sentido estricto. Se rechazó también la idea de introducir clínica jurídica. ${ }^{82}$ Uno de los estudiantes, Roger Wood, recordaba luego que no se discutían asuntos de justicia ni el lugar del sistema jurídico en la sociedad. ${ }^{83}$ En líneas generales, la orientación era langdelliana.

Las relaciones entre los profesores y estudiantes son recordadas como difíciles en el salón de clase y como amistosas fuera de él. Los 150 admitidos formaban una sola sección. Para comenzar, los profesores les indicaban que sólo esperaban que la mitad de la clase se graduara, y ponían

81 En el lenguaje de la Escuela de Derecho de Stanford lecturer es una persona que enseña, pero no es reconocido formalmente como professor. Con frecuencia son de tiempo parcial.

82 Lempert, op. cit., p. 270

83 Ibidem, p. 269

Esta obra está bajo una Licencia Creative Commons Atribución-NoComercial-SinDerivar 4.0 Internacional, IIJ-UNAM. Boletín Mexicano de Derecho Comparado, núm. 151, pp. 313-358. 
mucha presión. George Osborne es descrito como un terror socrático y un tirano; Schumway, como ácido y cascarrabias. ${ }^{84}$ La tensión se agravaba, porque todos los cursos se aprobaban con exámenes, y éstos eran todos en mayo. Era el estilo harvardiano.

Ente 1946 y 1962, el decano fue Carl B. Spaeth, quien tenía una excelente formación intelectual y una vocación internacionalista. Entre 1940 y 1946 había trabajado en el Departamento de Estado a cargo de las relaciones con América Latina. Bajo su liderazgo se crearon las primeras tres revistas académicas de derecho, y buscó recursos para la escuela. Spaeth introdujo cursos en derecho público y derecho internacional. Introdujo también cursos basados en problemas.

Sin duda, la política de contratación de profesores del decano Spaeth y luego del decano Manning explica el cambio de la escuela desde el punto de vista de lo que ofrecía. En 1953, la escuela contrató a John Henry Merryman, ${ }^{85}$ hasta entonces un joven profesor en Santa Clara, quien se distinguió luego por sus rigurosos y novedosos trabajos de investigación y su vocación internacional. En 1962, Spaeth realizó el llamado Columbia raid: la contratación de cinco profesores de la Escuela de Derecho de Columbia. Nótese que esto implicó una inversión de la tendencia anterior. Ahora profesores que se distinguían en otras escuelas de derecho eran contratados por Stanford. Lawrence Friedman, quien era ya una de las estrellas de la historia del derecho y del naciente campo de la moderna sociología jurídica, se incorporó a Stanford desde Wisconsin en 1968. Esto explica al menos una parte de la transformación de la Escuela de Derecho.

En 1975, la Escuela de Derecho era muy distinta a la de 1950. Ese año académico las actividades se iniciaron en un edificio nuevo, el primero diseñado especialmente para la escuela. El Program of Study 19751976 contiene varias fotografias del edificio y de las nuevas facilidades educativas que contiene. Informa que tiene una biblioteca de 260,000

$84 \quad$ Ibidem, p. 268.

85 John Henry Merryman (1920-2015), JD de Notre Dame y LLM y SJD New York University. Para una entrevista biográfica y su contribución al derecho comparado (Pérez Perdomo, Rogelio, "Conversación con John Henry Merryman sobre la investigación en derecho comparado en los Estados Unidos", Boletín Mexicano de Derecho Comparado, vol. 44, núm. 132, 2011). Merryman creó un nuevo campo en el derecho: propiedad cultural (Shapiro, Daniel, "A Universalist: Fathering Fields", International fournal of Cultural Property, 21, 2014, pp. 237-242).

Esta obra está bajo una Licencia Creative Commons

Atribución-NoComercial-SinDerivar 4.0 Internacional, IIJ-UNAM.

Boletín Mexicano de Derecho Comparado, núm. 151, pp. 313-358. 
volúmenes, y que el edificio tiene capacidad para albergar 450,000. ${ }^{86}$ Los profesores estaban tan orgullosos de su nuevo edificio, que publicaron un conjunto de trabajos que daban muestra de sus investigaciones. ${ }^{87}$ Esta era la nueva faceta: los profesores se pensaban a sí mismos como profesoresinvestigadores.

La lista del personal enseñante en 1975 es también diferente: seis profesores eméritos, lo que revela que la escuela retenía a los profesores hasta su retiro y aún más allá; ${ }^{88}$ treinta profesores y siete profesores asistentes o asociados; siete profesores visitantes; diez lecturers, y seis teaching fellows. Entre los profesores aparecen, además de Merryman y Friedman, ya mencionados, Mauro Cappelletti, ${ }^{89}$ comparatista, que era profesor en Florencia y Stanford; Víctor H. Li, un connotado especialista en derecho chino; Jane Collier, con designaciones en antropología y derecho; Yosal Rogat, compartido con el departamento de ciencias políticas, y Donald Lunde, profesor de psiquiatría, que colaboraba con la clínica y se interesaba en derecho y ciencias de la conducta. Aparece también Barbara A. Babcock, la primera mujer profesora, y William B. Gould, el primer profesor de ascendencia africana (derecho del trabajo).

El Program of Study 1975-1976 hace muy claro el cambio de orientación: el interés está en desarrollar las destrezas básicas del abogado: la escritura y lo básico de la investigación. Destaca la importancia del contexto económico y social para la comprensión del derecho, lo mismo que su dimensión ética. El listado de asignaturas del primer año, ahora semestrales, se ha simplificado. Están los cursos tradicionales: contratos, responsa-

86 Stanford Law School (1975), Stanford Law School. Program of Study 1975-1976, Stanford, p. 7.

87 Merryman, John Henry (ed.), Stanford Legal Essays, Stanford, Stanford University Press, 1975.

88 Los profesores eméritos pueden continuar enseñando con base en contratos por curso, y en caso de hacerlo mantienen su oficina.

89 Mauro Cappelletti (1927-2004). Graduado en derecho en Florencia. Fue luego profesor en Macerata, Friburgo, Universidad de Florencia, y presidio el Departamento de Derecho del European University Institute. Fue profesor en la Escuela de Derecho de Stanford a partir de 1970, y declarado emérito en 1996. Fue un procesalista muy reconocido, e incorporó la investigación política y social en el derecho comparado. Para su biografía intelectual: Ferrer Mac-Gregor, Eduardo, "Mauro Cappelletti y el derecho procesal constitucional comparado", Anuario Iberoamericano de Fusticia Constitucional, 13, 2009, pp. 267-306. 
bilidad extracontractual, procedimiento civil, propiedad, y se han incorporado derecho constitucional y derecho penal. Se espera que el primer año comunique la estructura básica del derecho de los Estados Unidos. Incluye también en investigación, bibliografía y escritura.

En los cuatro siguientes semestres los estudiantes tienen una oferta amplia de cursos en distintos campos, que no tienen un orden de prelaciones. Además de cursos con los nuevos desarrollos del derecho, como antitrust, derecho administrativo, instituciones bancarias, derecho ambiental, protección del consumidor, taller en problemas de derecho constitucional y pruebas, se ofrecen cursos como derecho chino, sistema jurídico soviético, derechos de Europa occidental y América Latina, adjudicación constitucional comparada, comparación de derecho de patentes y copyright, historia del derecho de los Estados Unidos, historia de la justicia criminal, antropología del derecho, controles internacionales del uso de fuerzas armadas, derecho y análisis microeconómico, industrias reguladas, derecho y política del gobierno urbano, petróleo y minería, filosofía del derecho y responsabilidad profesional. El listado no es exhaustivo.

Los cursos se hicieron más pequeños. Sin duda esto tenía relación con la disponibilidad de profesores y con las preferencias de éstos. En el primer semestre el curso de procedimiento civil se dividió en cinco secciones; el de contratos, en tres; el de derecho criminal, en dos; el de responsabilidad extracontractual, en tres. En el segundo semestre, el procedimiento civil II tenía dos secciones; derecho constitucional, cuatro; contratos II, tres secciones, y propiedad, dos secciones. Para los cursos superiores cada profesor fijaba el tope de estudiantes que aceptaba.

Las obras didácticas usadas en la Escuela de Derecho de Stanford, y también en otras universidades, muestran la transformación del pensamiento jurídico. Siguen siendo libros de casos, y siguen abundando las decisiones judiciales; pero ahora incluyen materiales de otras disciplinas, informaciones periodísticas y, en general, apoyos para entender los casos y problemas en su contexto social, económico o político. Esto ha implicado también cambios en el papel del profesor. El profesor socrático ha dado paso a un profesor que explica más y que promueve una discusión más distendida entre los estudiantes. El propósito ya no es que el estudiante descubra los principios jurídicos ínsitos en las decisiones de los casos, sino que entienda los casos en su contexto y que aprecie las políticas públicas o las opciones de valor que implican las decisiones. Para este tipo de 
discusiones, que son más de tipo seminario, los cursos reducidos son más funcionales.

Entre la literatura didáctica publicada por profesores de la Escuela de Derecho encontré A Primer on American Labor Law (Gould, 2013). Es una obra muy similar a un manual usado en las escuelas de derecho tradicionales de la América Latina y de Europa occidental. ${ }^{90}$ Es una obra que no está dirigida a los estudiantes de derecho, sino a otros estudiantes, sindicalistas y público general, para informarlos de las reglas principales del derecho laboral de los Estados Unidos. El profesor Gould me indicó que informa de su existencia a los estudiantes de derecho, pero que la obra didáctica que usó hasta su retiro fue el libro de casos de Heinz, Nolan \& Bales (2002).

El sistema de evaluación también cambió, y varía según los cursos. En algunos se podía exigir examen; en otros, un trabajo escrito. La escuela estableció lo que consideraba buenas prácticas en evaluación. El hacer todas las evaluaciones con exámenes y concentrarlas en mayo fue abandonado

El cuerpo estudiantil también cambió sustancialmente. El Program of Study 1975-1976 advierte que ingresaban quienes obtenían un alto puntaje en el LSAT, ${ }^{91}$ pero señala que los estudiantes provenientes de minorías étnicas tienen un estudio más pormenorizado de sus credenciales. El documento no lo dice, pero la lista de admitidos sugiere que los estudios de pregrado en una institución muy acreditada eran influyentes. La procedencia geográfica también cambió. De los 160 previstos para graduarse en 1975 como JD, sólo 51 declaraban a California como residencia de origen. Esto implica que la Escuela de Derecho se había convertido en nacional, pues 106 venían de otros estados de los Estados Unidos. De manera gruesa esta composición se mantuvo hasta el final del periodo que analizamos. En 1975 sólo tres venían del extranjero. Había dos estudiantes de máster, ambos de Florida, y cinco de doctorado: dos franceses, un

90 El estilo es similar, pero el contenido es diferente a los manuales de derecho del trabajo: introducción histórica y desarrollo de la legislación laboral/prácticas laborales injustas/negociación colectiva (incluyendo el contexto económico y estrategias de negociación)/solución de disputas/sector público. Las reglas laborales se ponen en un contexto muy práctico y actualizado.

91 El LSAT es un examen que mide el apresto para estudiar derecho. No es administrado por las universidades individualmente. Al contrario, que los admitidos tengan un alto puntaje en ese examen incide en la clasificación de la escuela. 
surcoreano, un japonés y un chino. Tanto el máster como el doctorado no estaban realmente organizados: implicaban un tiempo de residencia en Stanford, un número de créditos a aprobar y un trabajo de investigación personal (tesis) bajo la dirección de uno de los profesores.

En definitiva, en este periodo la Escuela de Derecho de Stanford se convirtió en una escuela de alcance nacional, que dio cabida a las nuevas corrientes del estudio interdisciplinario del derecho. Había claramente una orientación hacia el derecho comparado: los cursos de Merryman, Cappelletti, Li, Collier, y Markovits, muestran esa vocación internacional de la escuela. También se desarrollaron programas dentro del movimiento de derecho y desarrollo. En la década de 1960, la escuela recibió a un grupo de funcionarios públicos de la India dentro de la idea del Estadoadministrador. ${ }^{92}$ Lawrence F. Ebb ${ }^{93}$ era el profesor-director del programa internacional de la Escuela de Derecho, con especial interés en India. En la de 1970 se desarrolló el llamado Chile program, que llevó a un grupo de jóvenes profesores chilenos interesados en desarrollar casos a estadías como visiting scholars. ${ }^{94}$ Por último, Merryman, ${ }^{95}$ con la colaboración de Friedman, Clark e investigadores de Europa y América Latina, desarrolló una ambiciosa investigación comparativa, que puso juntos indicadores de cambio. En 1983, Barton, Gibbs, Li, \& Merryman publicaron un volumen colectivo sobre el derecho en culturas radicalmente diferentes..$^{96}$ Todo esto mostraba la vocación internacional o globalizadora que se percibía antes de 1995, aunque el público al cual se dirigía fuera principalmente de los Estados Unidos.

En la década de 1990, las cartas anuales que dirigía el decano Paul Brest a los egresados y a los profesores muestran la enorme atención que se

92 Información del profesor Marc Galanter, quien actuó como coordinador del grupo. Conversación personal en 2015.

93 Lawrence Foster Ebb (1918-2000) era un graduado con honores de Harvard en 1946. Fue clerk en la Corte Suprema de los Estados Unidos. Fue profesor de derecho y director del Stanford International Legal Studies 1954-1964. Salió de Stanford para ser consultor jurídico de General Motors y luego árbitro reconocido.

94 Merryman, John Henry, "Law and Development Memoirs I: The Chile Law Program", American fournal of Comparative Law, 48, 2000.

95 Merryman, John Henry (ed.), Law and Social Change in Mediterranean Europe and Latin America: A Handbook of Legal and Social Indicators for Comparative Study, Stanford, Stanford Law School, 1979.

96 Barton, J. H. et al., Law in Radically Different Cultures, St Paul, West Publishing, 1983.

Esta obra está bajo una Licencia Creative Commons

Atribución-NoComercial-SinDerivar 4.0 Internacional, IIJ-UNAM.

Boletín Mexicano de Derecho Comparado, núm. 151, pp. 313-358. 
prestaba a la contratación de profesores, al financiamiento y a la orientación de la educación. Las cartas muestran que el patrón de contratación de profesores era atraer a profesores jóvenes que se destacaban como líderes en su campo en otras escuelas de derecho. El financiamiento adicional a la matrícula estudiantil se veía como imprescindible para pagar salarios competitivos con escuelas más acreditadas. En cuanto a la orientación, había la decisión de no hacer mayores cambios en el primer año, por considerar que el método de casos proveía competencias analíticas invalorables, pero otras competencias eran también importantes. Las cartas mencionan la competencia de comunicación escrita: redacción de contratos, memorandos e informes. También competencias cuantitativas para análisis económico, estadístico, financiero y de contabilidad. La escuela también se ocupaba de proveer destrezas para la negociación, el consejo, la solución de conflictos, la toma de decisiones. Dentro de la escuela se creó un grupo de trabajo para evaluar los logros en el desarrollo de esas competencias.

\section{3. ¿Una Escuela de Derecho para un mundo globalizado?}

En el trimestre del otoño de 2016 la Escuela de Derecho muestra una población estudiantil diferente a la de 1975. La escuela sigue admitiendo 180 candidatos por año al JD, además de una docena de transferencias de otras universidades de prestigio similar. Esto hace una población total de candidatos al JD de unas 550 a 560 personas. El 33\% viene del Far West (California, Nevada y Hawái). El 3\% viene del extranjero. Además, recibe unos ochenta candidatos a los diferentes programas de máster (un año de duración ${ }^{97}$ y unas cinco personas como candidatos a doctorado (SJD), que es un programa de varios años. Casi todos los cursantes de máster y SJD son extranjeros. En total, la población estudiantil de la escuela es de unas 670 personas ${ }^{98}$ y aproximadamente uno de

97 Los programas de máster se indican a continuación con el número de admitidos en 2016 entre paréntesis: Corporate governance and practice (21), Environement Law and Policy (13), Law, Science and Technology (22), International Economic Law, Business and Politics (15), Stanford Program for International Legal Studies (SPILS, que es el máster de investigación, 12). Candidatos doctorales eran 27 en octubre 2016. Información de la Escuela de Derecho.

98 Durante el decanato de Larry Kramer (2004-2012) la Escuela de Derecho optó por periodos académicos trimestrales usados en el resto de la universidad para facilitar que estu- 
cinco viene del extranjero, y uno de cada cuatro, de California. Es una escuela nacional con una fuerte presencia internacional.

El incremento del número de estudiantes se ha visto más que compensado por el del cuerpo profesoral. El folleto publicitario de la Escuela de Derecho informa que la faculty tiene un tamaño de ochenta, y que la relación de estudiantes por profesor es de 7.3 a 1. En el primer año el tamaño usual de las secciones es de treinta. En los cursos del segundo y tercer año, que son usualmente electivos, el tamaño es generalmente más reducido. El 70\% de los cursos tiene veinte estudiantes o menos. Existen también grupos de discusión que tienen lugar en la casa de los profesores con un promedio de ocho estudiantes (Stanford Law School, 2016). El prestigio de Stanford le permite hacer una selección muy cuidadosa. No sólo vienen de excelentes estudios de pregrado (college), sino que frecuentemente tienen una experiencia vital o de trabajo, con alguna frecuencia fuera de los Estados Unidos.

La matrícula en la Escuela de Derecho es muy elevada. Para 20162017 alcanza a 56,000 dólares, y el costo total, incluyendo residencia y alimentación, es de 88,000. Sin embargo, la composición social es variada. La mayor parte de los estudiantes del programa JD reciben asistencia financiera (información de Internet y de la Escuela de Derecho). Dado el prestigio de la escuela, el pago de la matrícula es generalmente una buena inversión. Típicamente los egresados del JD son solicitados por las firmas de abogados más importantes de los Estados Unidos. En la lista de Am Law 100 law firms, que da datos sobre las cien firmas más importantes por sus ingresos en los Estados Unidos, los egresados de Stanford trabajan como socios en noventa de ellas, y como asociados, en 94. Los egresados de Stanford reciben como salario de inicio en esas firmas 160,000 dólares por año (Stanford Law School, 2016). Claramente estudiar derecho en Stanford es una inversión importante, que puede resultar productiva.

El interés por el derecho comparado, los derechos extranjeros y el derecho internacional no es sólo un asunto puramente intelectual. Los estudiantes de la Escuela de Derecho saben que probablemente van a lidiar con negocios internacionales, y Stanford se promueve como una Escuela de Derecho globalizada.

diantes de otras escuelas o departamentos puedan tomar cursos en derecho y que los estudiantes de derecho puedan tomar cursos fuera de los ofrecidos por la escuela.

Esta obra está bajo una Licencia Creative Commons

Atribución-NoComercial-SinDerivar 4.0 Internacional, IIJ-UNAM.

Boletín Mexicano de Derecho Comparado, núm. 151, pp. 313-358. 
La International Law Society, es decir, la asociación que agrupa a los estudiantes interesados en temas internacionales o en derechos extranjeros, tiene una membresía de unos ochenta JD. Uno de sus eventos anuales, la cena para que los estudiantes interesados en lo internacional conozcan a los profesores con cursos con dimensión internacional, fue concurrida por unos cien estudiantes y quince profesores, en octubre 2016.

En la actualidad, los estudiantes pueden participar en la Global initiative, que comprende tres elementos: un curso en global legal practice, cursos con estudios intensivos fuera del país, y la incorporación de elementos internacionales en cursos fundamentales de derecho. Global legal practice fue creado en 2014-2015. Prepara a los estudiantes para trabajar con clientes con asuntos interconectados entre derecho y negocios. Usa más el tipo de casos y discusiones de casos al estilo de las escuelas de negocios. Los casos son problemas reales de transacciones complejas. En el curso colaboran los consultores jurídicos de Google, Cisco y abogados de firmas internacionales.

Los estudiantes han viajado, según el periodo, a China, India, Brasil, Chile, y en Europa a ciudades como La Haya y París. Los viajes duran entre siete y diez días en periodos intertrimestrales. Los estudiantes también pueden pasar un periodo académico fuera (Foreign legal studies program) Bucerus Law School (Hamburgo), Hebrew National University of Jerusalem, Institut d'Études Politiques (Paris), National University of Singapore, Peking University Law School, Waseda University Law School.

El esfuerzo de proveer un currículo con acento en derecho comparado y temas internacionales era visible en la década de 1970, e indudablemente logró despertar la vocación hacia el derecho comparado de estudiantes que se convirtieron luego en académicos distinguidísimos en el área: David S. Clark (JD. 1969, JSM. 1972) y Bryant Garth (JD. 1975, PhD en el European University Institute de Florencia, 1979). Sin embargo, la Escuela de Derecho atraía muy escasos estudiantes extranjeros. El cambio se produjo con la creación del Stanford Program in International Legal Studies (SPILS). Este programa, pensado por los profesores Lawrence Friedman y Thomas Heller, es un programa dirigido a los estudios interdisciplinarios de derecho. Entre sus requisitos de admisión, además de los usuales para posgrado, está una experiencia de investigación. Cada año admite una docena de candidatos, que con frecuencia son jóvenes profesores o investigadores en sus países de origen. Sus ejes centrales son un seminario de 
derecho sociedad ${ }^{99}$ muy volcado al análisis de distintos aspectos de la vida del derecho en distintos países, y un seminario de metodología de la investigación empírica en el derecho, que desemboca en un taller de tesis. Además, los SPILS fellows deben tomar un número de créditos en la Escuela de Derecho y en otros departamentos de la Universidad. La orientación del programa es interdisciplinaria y dirigida a los estudios empíricos o de campo, con cierto acento en el análisis de la relación entre derecho y políticas públicas. El programa conduce a un máster de investigación (Scientia Furis Master - SJM). Buena parte de estas tesis han sido publicadas luego en distintas revistas tanto de Estados Unidos como de otros países.

La experiencia de SPILS resultó tan positiva, que se ha convertido en la única vía de ingreso al programa doctoral (SJD). La demanda de ingreso resultó tan alta, que la Escuela de Derecho decidió abrir otros programas de máster en derecho (LIM), más dirigidos a la formación profesional. Son también programas principalmente dirigidos a un público de graduados extranjeros. Tienen también un acento interdisciplinario, pero el componente de investigación no es tan fuerte. El programa de doctorado, SPILS y los másters en derecho son los responsables de la presencia significativa de más de cien estudiantes extranjeros en la Escuela de Derecho.

La composición del cuerpo profesoral también ha cambiado. Lo característico del cuerpo profesoral de hoy, comparado con el de 1975 o de 1950, es el número significativo de publicaciones por profesor. Muchas de estas publicaciones pueden ser clasificadas como interdisciplinarias (law and economics, law and society, etcétera). En la contratación de profesores de los últimos veinte años predomina la de profesores con un doctorado en una disciplina distinta al derecho, junto con un grado en derecho. $\mathrm{Ha}$ aumentado también la diversidad étnica y el número de profesoras. Una de ellas, la profesora asociada Shirin Sinnar, tiene además de grado en derecho de Stanford, un M.Phil de relaciones internacionales de Cambridge. Usa hijab islámico. Incluso la escuela ha tenido dos decanas en los últimos veinte años. Lo que no ha variado es la composición estadounidense del cuerpo profesoral. Sólo una profesora, Barbara van Schewick, es graduada fuera de los Estados Unidos. La mayor parte de los cursos de

99 El The Law and Society/SPILS Core Seminar es dirigido por Lawrence Friedman, con la colaboración de Rogelio Pérez-Perdomo. La orientación internacional y sociojurídica del seminario se ve reflejada en la obra de Lawrence Friedman, R. Pérez-Perdomo \& M. Gómez (eds.), Law in Many Societies, Stanford, Stanford University Press, 2011.

Esta obra está bajo una Licencia Creative Commons

Atribución-NoComercial-SinDerivar 4.0 Internacional, IIJ-UNAM.

Boletín Mexicano de Derecho Comparado, núm. 151, pp. 313-358. 
orientación internacional están a cargo de profesores que han realizado sus estudios exclusivamente en los Estados Unidos, de profesores visitantes o lecturers. Seguramente esto no se deba a un prejuicio colectivo, sino a la dificultad de evaluar a los colegas con formación en el extranjero y publicaciones en idiomas distintos al inglés. Como una parte importante del cuerpo profesoral sólo lee en inglés la exigencia de rigor, en la selección actúa como una limitante a una mayor variedad y cosmopolitismo del cuerpo profesoral.

\section{ALLÁ Y AQUí}

La historia de la educación jurídica en los Estados Unidos impresiona por el éxito mundial alcanzado en un tiempo relativamente breve. Cuando se analiza especialmente el caso de la Escuela de Derecho de Stanford, es todavía más impresionante: hacia 1950, una escuela modesta que sesenta o setenta años después ha alcanzado una posición de ser mundialmente reconocida como una de las más importantes y enormemente atractiva para graduados de todo el mundo. De allí el interés de estudiar estas instituciones.

La visión del comparatista puede ser útil, pues quienes están demasiado inmersos y no tienen referentes comparativos difícilmente pueden entender peculiaridades y sutilezas.

Un primer elemento a destacar es un factor obvio que impresiona al visitante latinoamericano o europeo: el dinero. Esto se refleja en los magníficos edificios, la calidad de las bibliotecas, la cantidad de recursos para la enseñanza y el aprendizaje, los pagos relativamente elevados a los profesores. Las escuelas de derecho de los Estados Unidos son enormemente ricas comparadas con las latinoamericanas o del continente europeo. Mucho de ese dinero viene de la habilidad para recibir donaciones importantes y cobrar matrículas elevadas a los estudiantes. No es este el lugar de entrar en mayores explicaciones: en un mundo que se mueve por incentivos materiales, una institución que no cuente con recursos para pagar bien a sus profesores, ofrecer recursos para la investigación y comodidades a todos, no puede ir demasiado lejos en la mejora de su calidad. Esta es una explicación que vale para las escuelas de derecho de los Estados Unidos en general, pero no explica las diferencias entre ellas: por qué unas ganan en prestigio y otras se hacen menos atractivas. La relación entre prestigio e ingresos es circular: 
las escuelas más prestigiosas son las que tienen mayor capacidad de lograr más fondos y a la vez la pérdida de prestigio generalmente implica una disminución de donaciones y otros ingresos.

El caso de la Escuela de Derecho de Stanford puede ayudar a comprender los mecanismos de ascenso. Al comienzo de la década de 1970, la escuela había consolidado el prestigio regional: era una escuela importante en el oeste de los Estados Unidos. El prestigio lo había alcanzado gracias a una política bien definida de contratar profesores que se hubieran distinguido por la calidad de sus publicaciones y de su enseñanza. En buena medida había seguido el liderazgo de Harvard, contratando graduados distinguidos de Harvard, pero había mirado también a profesores que se habían distinguido en otras escuelas. Pero hubo también nuevas políticas: a pesar de tener un público estudiantil predominantemente regional y nacional y muy dirigido a la práctica profesional, decidió acoger los estudios interdisciplinarios del derecho y renovar los métodos educativos y la oferta de cursos. En el momento que los profesores afiliados a los critical legal studies encontraban rechazo en Harvard o Yale por considerarlos disruptivos o amenazantes, Stanford no sentía esa amenaza. También profesores de law and society y law and economics eran acogidos. El elenco de profesores impresiona también por su apertura internacional, aunque se estaba todavía lejos de los tiempos de globalización. No era frecuente en la época, encontrar en otras escuelas de derecho, cursos sobre derecho italiano, chino o soviético, o contar con profesores interesados en la solución de conflictos en el sur de México o en países africanos. Cuando deciden abrirse a los estudiantes de fuera de los Estados Unidos no adoptan el tradicional curso de máster, sino que innova con un máster de investigación, y luego con másters orientados a nuevos desarrollos del derecho. La innovación más reciente es la expansión de los planes de estudios conjuntos, que permiten que los estudiantes integren conocimientos y capacidades diversas y multidisciplinarias. Esto es también muestra de este espíritu de innovación y apertura. El contraste con las escuelas latinoamericanas y europeas con planes de estudio rígidos, donde los profesores enseñan año tras año los mismos cursos, y donde los profesores no se replantean los temas y los métodos educativos, la diferencia es impresionante. La Escuela de Derecho de Stanford es en ese sentido un ejemplo a tener presente. 


\section{REFERENCIAS BIBLIOGRÁFICAS}

Aввотт, Nathan (1901), "The Undergraduate Study of Law", Annual Report American Bar Association, 24.

ABEL, Richard (1989), American Lawyers, New York, Oxford University Press. Association of American Law Schools, Directory of Teachers in Member Schools, disponible en: http://heinonline.org/HOL/Index?index=aals/ aalsdltEcollections $=$ aals.

Barton, John H. et al. (1983), Law in Radically Different Cultures, St Paul, West Publishing.

BernsteIn, Richard B. (2004), Thomas Fefferson: the Revolution of Ideas, New York, Oxford University Press.

Calhoun, Charles W. (2005), Benjamin Harrison, New York, Times Books.

CHase, Anthony (1979), "The Birth of the Modern Law School", The American Fournal of Legal History, vol. 23 núm. 4.

Cirigliano, Gustavo y Villaverde, A. (1967), Dinámica de grupos y educación; fundamentos y técnicas, Buenos Aires, Humanitas.

Ellickson, Robert (1997), "The Market for 'Law and' in Legal Scholarship”, Yale Law School. Faculty Scholarship Series. Paper 377, disponible en: http://digitalcommons.law.yale.edu/fss_papers.

Ellickson, Robert (2000), "Trends in Legal Scholarship: A Statistical Study", Journal of Legal Studies, vol. 29, núm. S1.

FERLING, John (1993), John Adams: A Life, New York, Oxford University Press.

Ferrer MaC-Gregor, Eduardo (2009), "Mauro Cappelletti y el derecho procesal constitutional comparado", Anuario Iberoamericano de Fusticia Constitucional, 13.

Friedman, Lawrence M. (2005), A History of American Law+, 3a. ed., New York, Simon \& Schuster.

Friedman, L., Pérez-Perdomo, R. y M. Gómez (eds.) (2011), Law in Many Societies, Stanford, Stanford University Press.

GEORGE, Tracey E. (2006), "An Empirical Study of Empirical Legal Scholarship: the Top Law Schools", Indiana Law Journal, vol. 81, núm. 1.

Gilmore, Grant (1977), Ages of American Law, New Haven, Yale University Press. 
GOEBEL Jr., Julius (1955), A History of the School of Law, Columbia University, New York, Columbia University Press.

Gordon, Robert W. (1988), "The Independence of Lawyers", Boston University Law Review, vol. 68, núm. 1.

Gordon, Robert W. (1989), "Critical Legal Studies as a Teaching Method", Legal Education Review, vol. 1.

Gordon, Robert W. (1995), "The Case for (and Against) Harvard", Michigan Law Review, vol. 93, núm. 6.

Gordon, Robert W (2004), "Professors and Policy Makers: Yale Law Faculty in the New Deal and After". Kronan, A. T. (ed.), History of the Yale Law School. The Tercentennial Lectures, New Haven, Yale University Press.

Gordon, Robert W. (2007), "The Geological Strata of the Law School Curriculum", Vanderbilt Law Review, 60.

Gould IV, William B. (2013), A Primer on American Labor Law, 5a. ed., Cambridge, Cambridge University Press (1a. ed. de 1982, Cambridge, MIT Press).

GRAY, John C. (1908), Select Cases and Other Authorities on the Law of Property, Cambridge, George H. Kent.

Grey, Thomas G. (1983), "Langdell's Orthodoxy", University of Pittsburgh Law School, 45.

Heinz, T., Nolan, D. \& BaLes, R. (2002), Labor Law, Collective Bargaining in a Free Society. 5a. ed., St Paul. West Group (la primera edición es de 1972 y los autores eran W. Oberer an K. Hanslowe).

Holmes, Oliver (1880), "Book Notices: Langdell and Anson on Contract", American Law Review, 14. Reproducido LEVINE, Martin L. (ed.), 1993, Legal education, New York, New York University Press.

Hurst, William (1956), Law and the Conditions of Freedom in Nineteenth Century United States, Madison, University of Wisconsin Press.

KaLman, Laura (1986), Legal Realism at Yale 1927-1960, Chapel Hill, University of North Carolina Press.

Kennedy, Duncan (1970-1971), "How the Law School Fails: A Polemic", Tale Review of Law and Social Action, 1.

KenNedy, Duncan (2004), Legal Education and the Reproduction Hierarchy, New York, New York University Press. 
Kimbalt, Bruce (2006), "The Proliferation of Case Method Teaching in American Law Schools, Mr. Langdell's Emblematic 'Abomination', 1890-1915". History of Education Quartely, vol. 46, núm. 2.

Kimbald, Bruce (2009), The Inception Of Modern Professional Education: C.C. Langdell, 1826-1909, Chapel Hill, University of North Carolina Press.

Kirkwood, Marion A. \& Owens, W. B. (1961), A Brief History of Stanford Law School, 1893-1896, disponible en: https//wrere.cdn.law.stanford.edu.

Konig, David T. (2012), "Thomas Jefferson and the Practice of Law", Encyclopedia Virginia, Virginia Foundation for the Humanities, Charlottesville.

LANGBEIN, John H. (2004), "Blackstone, Lichtfield and Yale: the Founding of Yale Law School", en Kronman, A. T. (ed.), History of the Yale Law School. The Tercentennial Lectures, New Haven, Yale University Press.

LangbeIn, John H (2004), "Law School in a University: Yale Distinctive Path in the Late Nineteenth Century", en Kronman, A. T. (ed.), History of the Yale Law School. The Tercentennial Lectures, New Haven, Yale University Press.

LANGDELL, Christopher Columbus (1887), "Harvard Celebration Speech", Law Quartely Review, 3. Consultado en Levine, Martin L. (ed.), 1993, Legal Education, New York. New York University Press.

LAPIANA, William P. (1994), Logic and Experience: The Origins of Modern American Legal Education, New York, Oxford University Press.

LEMPERT, David (2003), "After Five Decades: Stanford Law School class of 1952”, Legal Studies Forum, 27.

LEVACK, Brian (1973), The Civil Lawyers in England, 1603-1641: a Political Study, Oxford, Clarendon Press.

Levine, Martin L. (ed.) (1993), Legal Education, New York, New York University Press.

Manning, Bayless (2008), Bayless Manning. Reflections on his Years at Stanford Law School, Interviewed by Sarah F. Wilson, Feb 18, 2008, Stanford Law School.

MaKenna, Marian (1986), Tapping Reeve and the Litchfield Law School, New York, Oceana.

MARQUIS who's who in American law (1a. ed., Chicago, 1977. Varias ediciones posteriores). 
Merryman, John Henry (ed.) (1975), Stanford Legal Essays, Stanford, Stanford University Press.

Merryman, John Henry (ed.) (1979), Law and Social Change in Mediterranean Europe and Latin America: A Handbook of Legal and Social Indicators for Comparative Study, Stanford, Stanford Law School, 1979.

Merryman, John Henry (2000), "Law and Development Memoirs I: The Chile Law Program", American Journal of Comparative Law, 48.

Merryman, John Henry \& Pérez Perdomo, Rogelio, The Givil Law Tradition, 3a. ed., Stanford, Stanford University Press, 2007.

MerTZ, Elizabeth (2007), The Language of The Law School: Learning To "Think Like a Laweyer", Oxford, Oxford University Press.

Moran, Gerald P. (2010), John Chipman Gray. The Harvard Brahmin of Property Law, Durham, Carolina Academic Press.

Osborn, John Jay (1971), The Paper Chase. Boston, Houghton Mifflin.

PACKer, Herbert \& EHRLICH, Thomas (1972), New Directions in Legal Education, New York, McGraw-Hill.

PÉrez Lledó, Juan A. (1992), "La enseñanza del derecho en los Estados Unidos", Doxa, núm. 12. El artículo fue también publicado en PÉREZLLEDÓ, J. A. (2006), La enseñanza del derecho / Dos modelos y una propuesta, Lima, Palestra; Bogotá, Temis.

PÉrez Lledó, Juan A. (1996), El movimiento Critical Legal Studies, Madrid, Tecnos.

PÉREZ-PEÑA, Richard (2014), "To Young Minds of Today, Harvard is the Stanford of the East", New York Times, May 29.

Pérez Perdomo, Rogelio (2011), "Conversación con John Henry Merryman sobre la investigación en derecho comparado en los Estados Unidos", Boletín Mexicano de Derecho Comparado, núm. 132.

Posner, Richard, "Legal Scholarship Today”, Stanford Law Review, vol. 45, núm 6, 1993.

Posner, Richard (ed.) (1992), The Essential Holmes, Chicago, University of Chicago Press.

Pound, Roscoe (1951), "Some Comments on Law Teachers and Law Teaching", Journal of Legal Education, 3.

PREST, Wilfrid (2008), William Blackstone: Law and Letters in the Eighteen Century, Oxford, Oxford University Press.

Esta obra está bajo una Licencia Creative Commons

Atribución-NoComercial-SinDerivar 4.0 Internacional, IIJ-UNAM.

Boletín Mexicano de Derecho Comparado, núm. 151, pp. 313-358. 
RADERMACHER, Ludger (1999), "The Stanford Program for International Legal Studies (SPILS) - An Alternative to the «Classical» LIM Program”, European Fournal of Law Reform, vol. 1, núm. 1-2.

REED, Alfred Z. (1928), Present Day Law Schools in the United States and Canada, New York, The Carnegie Foundation for the Advancement of Sciences.

REHNQUiST, William (1986), "The Lawyer-Statesman In American History", Harvard Fournal of Law and Policy, 9.

Sauder, Michael \& Lancaster, R. (2006), "Do Rankings Matter? The Effect of US News and World Report Rankings on the Admission Process of Law Schools", Law and Society Review, vol. 40. núm. 1.

Schlegel, John H. (1995), American Legal Realism and Empirical Social Science, Chapel Hill, University of North Carolina Press.

Schuck, Peter H. (1989), "Why don't Law Professors do More Empirical Research?", Fournal of Legal Education, vol. 39, núm. 3.

SHAPIRO, Daniel (2014), "A Universalist: Fathering Fields", International Fournal of Cultural Property, vol. 21, núm. 3.

Stanford Law SchoOL (sf): 60+ Moments in the History of Stanford Law School. Exhibition at Stanford Law School, disponible en: https//law.stanford.edu/about/history.

STANFORD LAW SchOOL (2016), Consider what Counts (folleto publicitario con resumen de las cifras).

Stanford LaW School (1975), Stanford Law School. Program of Study 19751976. Stanford.

Stevens, Robert (1983), Law School. Legal Education in America from 1850s to the 1980s, Chapel Hill, The University of North Carolina Press.

Stone, Harlan F. (1941), "Nathan Abbott", Columbia Law Review, XLI, núm. 6.

Sullivan, W. M. et al. (2007), Educating Lawyers / Preparation for the Profession of Law, San Francisco, John Wiley.

Tocoueville, Alexis de (1835-1840), La démocratie en Amérique, París, J. Vrin, 1990.

Touraine, Alain (1974), The Academic System in American society, New York, McGraw-Hill.

Turow, Scott (1977), One L., New York, Putnam. 
Tushnet, Mark (1987), "Legal Scholarship in the United States: An Overview", Modern Law Review, 50, núm. 6.

Warren, Charles (1912), A History of American Bar, London, Cambridge University Press.

Watson, Allan (2006), The Shame of American Legal Education, Lake Mary, FL, Vandepas Publishing. 